\title{
ANÁLISE PREQÜENCIAL
}

\author{
Airlane Pereira Alencar
}

DISSERTAÇÃO APRESENTADA

$\mathrm{AO}$

INSTITUTO DE MATEMÁTICA E ESTATÍSTICA

DA

UNIVERSIDADE DE SÃO PAULO

PARA OBTENÇÃO DO GRAU

$\mathrm{DE}$

MESTRE EM ESTATÍSTICA

\section{Área de Concentração: Estatística \\ Orientador: Prof. Dr. Sergio Wechsler}

Este trabalho foi financiado pela

Fundação de Apoio á Pesquisa do Estado de São Paulo

São P. '? dezembro de 2000 - 


\section{ANÁLISE PREQÜENCIAL}

Este exemplar corresponde à redação final da dissertação devidamente corrigida e defendida por Airlane Pereira Alencar e aprovada pela comissão julgadora.

São Paulo, 29 de dezembro de 2000.

Banco Examinadora:

- Prof. Dr. Sergio Wechsler (Orientador) - IME - USP

- Prof. Dr. Carlos Alberto de Bragança Pereira - IME - USP

- Profa. Dra. Lurdes Inoue - University of Texas 


\section{Agradecimentos}

Gostaria de agradecer a todos que me ajudaram na realização deste trabalho. Primeiramente, minha família que sempre me apoiou desde o início. Ao Sergio, que aceitou me orientar quando fui bater em sua porta. À Delhi, ao Henry e ao Alberto por contribuirem diretamente na preparação desta dissertação e por sua amizade. Aos professores do IME, que sempre estiveram dispostos a me ajudar, em especial à profa. Clelia, pois sempre me permitiu invadir sua sala, e ao prof. Antonio Carlos, que me ajudou na aplicação que envolve análise de sobrevivência. À profa. Lurdes quie veio direto do aeroporto para participar da banca e ao prof. Carlos Alberto por aceitar participar tão prontamente participar de emergência. Obrigada a todos os meus amigos! 


\section{Resumo}

Basicamente, a análise preqüencial consiste na produção e avaliação de previsões para quantidades observáveis. Por previsão, entende-se uma distribuição de probabilidades para a quantidade desconhecida de interesse. Assim definida cada previsão é coerente segundo a noção de coerência de De Finetti.

Neste trabalho são estudados sistemas de previsão e diversas medidas de avaliação, incluindo o comportamento assintótico destas medidas para um bom sistema de avaliação. Neste contexto, são apresentados os princípios preqüenciais. Por fim, são apresentadas quatro aplicações analisadas sob a ótica preqüencial. 


\section{Abstract}

Prequential Analysis consists of the production and evaluation of previsions for observables quantities. We mean by prevision a probability distribution for the quantity of interest. So defined, each prevision is coherent in de Finetti's sense.

In this work we study prevision systems and several evaluation measures, including the asymptotic behavior of these measures for a good prevision system. In this context, the prequential principles are presented. Finally, four real data applications are developed under the prequential point-of view. 


\section{Sumário}

1 Introdução 1

2 Coerência $\quad 3$

2.1 Coerência Temporal e Coletiva . . . . . . . . . . . . . . . . 4

3 Sistemas de Previsão $\quad 7$

4 Avaliação do sistema de previsão $\quad 12$

4.1 Tipos de medidas de avaliação . . . . . . . . . . . . . . . . . . 13

4.2 Princípio Forte Preqüencial f . . . . . . . . . . . . . . . . . . . 14

4.3 Calibração . . . . . . . . . . . . . . . . . . 15

4.4 Medida escalar de confiabilidade e Escore de

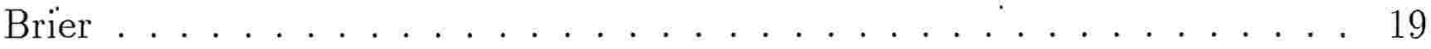

4.5 Transformada da integral de probabilidade . . . . . . . . . . . 20

4.6 Funções de perda . . . . . . . . . . . . . . . . . . . . . 21

4.7 Escore de Brier e medida de De Finetti . . . . . . . . . . . . . . . . . 23

4.8 Verossimilhança Preqüencial . . . . . . . . . . . . . . . 24

4.9 Consistência e Eficiência Preqüencial . . . . . . . . . . . . 25

5 Considerações sobre a implementação da Análise Preqüencial 27

5.1 Escolha de modelos . . . . . . . . . . . . . . . . . . . . . 28

5.2 Escolha do sistema de previsão . . . . . . . . . . . . . . . . 29 
7 Aplicações $\quad 33$

7.1 Índice de mortalidade infantil . . . . . . . . . . . . . . . . . . 33

7.2 Modelagem de contornos acentuais no português . . . . . . . . . . 37

7.3 Sobrevivência após o diagnóstico de mieloma . . . . . . . . . . . . . 39

7.4 Séries temporais com modelos autoregressivos . . . . . . . . . . . . . 41

8 Conclusões $\quad 47$ 


\section{Capítulo 1}

\section{Introdução}

Uma das maiores preocupações da Inferência Estatística, e da Ciência como um todo, é avaliar continuamente seus métodos e modelos. A postura subjetivista de Bruno de Finetti (1974) de indução particulariza-se, no caso da Inferência Estatística, em uma inferência bayesiana operacionalista. Isto significa que a Inferência Estatística é entendida como a construção de previsões probabilísticas e pessoais a respeito de quantidades de valor desconhecido julgadas relevantes, utilizadas as informações disponíveis. Mais ainda, tais quantidades devem sempre ser potencialmente observáveis, de tal modo que qualquer afirmação sobre seu valor sempre possa posteriormente ter verificada sua veracidade de forma inequívoca.

A análise preqüencial incorpora a esta atitude a preocupação com a avaliação das previsões realizadas. Estas avaliações, por sua vez, devem depender exclusivamente das previsões anunciadas e dos valores das variáveis efetivamente observados. A disponibilidade destes valores é garantida justamente pela postura operacionalista descrita no parágrafo anterior.

A análise preqüencial sugere a análise dos dados de modo seqüencial. Considere, então, a seqüência $y^{(n)}=\left\{y_{1}, \ldots, y_{n}\right\}$. Em cada instante $i(i=1, \ldots, n)$, pode-se utilizar $y^{(i)}$ para produzir uma previsão para a próxima observação e compará-la com seu valor efetivamente observado, $y_{i+1}$.

A análise preqüencial consiste, portanto, na produção seqüencial de previsões para 
quantidades ainda não observadas e na avaliação - também seqüencial - destas previsões. Estas quantidades não observadas são desconhecidas e, portanto, constituem um conjunto de variáveis aleatórias. Deste modo, uma previsão coerente, que visa quantificar a incerteza acerca desta variável, consiste numa distribuição de probabilidades (ver Capítulo 2).

Devido a este caráter seqüencial da análise e consistir esta em produção e avaliação de previsões, esta nova abordagem chama-se preqüencial, tendo sido construída e desenvolvida por A. P. Dawid (1984, 1992, 1997, 1999).

Um aspecto fundamental da análise preqüencial é que a avaliação dos métodos de previsão deve ser feita exclusivamente com base nas previsões e nos dados observados correspondentes a estas previsões, sem a dependência de quantidades desconhecidas ou não observadas. Esta característica define o Princípio Preqüencial.

Em toda análise cujos dados apresentam uma ordenação natural, a análise preqüencial segue com a produção seqüencial de previsões e suas avaliações. Em situações em que não há esta ordenação da amostra de dados de tamanho $N$, existem $N$ ! seqüências ordenadas correspondentes a esta amostra. Se julgamentos de invariância quanto à ordenação dos dados forem assumidos, e conseqüentemente, se os modelos propostos para estes dados não levam em consideração sua ordem, então parece bastante razoável escolher qualquer ordem "ao acaso" para os dados (uma ordem que de preferência não seja resultado da ordenação segundo uma variável do problema), e proceder a análise preqüencial para avaliar o modelo envolvido na análise. Assim, mesmo problemas sem uma ordenação natural e sem objetivos preditivos podem ser analisadas sob a ótica preqüencial apenas para que sejam avaliados os modelos envolvidos em suas análises. Para tal avaliação, estes modelos devem ser utilizados na construção das previsões.

Enfim, a análise preqüencial fornece um novo modo de tratar os mais variados problemas estatísticos, e não somente os de previsão, com ênfase na avaliação de tais métodos com base nos dados disponíveis. 


\section{Capítulo 2}

\section{Coerência}

Considere a construção de probabilidade subjetiva baseada em apostas proposta por Bruno de Finetti (de Finetti (1931) e Loschi (1992)).

Um cenário de apostas representa qualquer situação em que um indivíduo está diante de uma quantidade $\Theta$ de valor desconhecido e necessita fornecer previsões, tirar conclusões ou tomar decisões que dependam de tal quantidade. A noção de coerência é adotada como um axioma de comportamento em tais situações, em particular na inferência estatística.

Para eventos $E$ a respeito do valor de $\Theta$, o indivíduo é forçado a anunciar taxas $P(E)$ para bilhetes que valerão $S_{E}$ caso $E$ ocorra ou zero, caso contrário. Estes anúncios são feitos sem que o indivíduo conheça os valores de $S_{E}$ a serem fixados pelo bookmaker (que conhece $P(E)$ ). Ao anunciar $P(E)$, o indivíduo se compromete a "comprar" bilhetes $E$ por $P(E) S_{E}$ (caso $S_{E}$ seja negativo, trata-se de uma venda).

Nesta situação, o melhor que o indivíduo pode fazer é anunciar taxas $P(E)$ de modo a não tornar seu lucro líquido certamente negativo após a revelação do valor de $\Theta$. Esta é a definição de coerência e o célebre teorema de B. de Finetti mostra que o indivíduo é coerente se, e somente se, $P(\cdot)$ é uma medida de probabilidade finitamente aditiva.

Um esquema de apostas semelhantes, mas que serão canceladas caso não ocorra o evento $\mathrm{F}$, implica a probabilidade condicional $P(E \mid F)=P(E, F) / P(F)$.

$\mathrm{Na}$ análise preqüencial, uma previsão de $Y_{n+1}$ constitui uma distribuição de probabilidades sendo, portanto, coerente. Note-se que esta coerência é estática, ou seja, para cada 
instante $n$ é satisfeito o axioma de coerência.

\subsection{Coerência Temporal e Coletiva}

Como extensão natural da noção de coerência estática, pode-se pensar numa formulação de coerência temporal obtida a partir da realização de eventos condicionais visando a atualização de uma previsão.

Suponha que no instante $t$ uma previsão (opinião ou probabilidade) $P_{t}(E)$ seja produzida para o evento $E$ e no instante $t^{*}(>t)$ deseja-se apresentar uma nova previsão $P_{t^{*}}(E)$ para $E$ devido à ocorrência de um evento $F$ previamente considerado.

O procedimento de Condicionamento Bayesiano propõe a atualização de $P_{t}(E)$ como $P_{t^{*}}(E)=P_{t}(E \mid F)$. Esta atualização é equivalente a supor $P_{t^{*}}(F)=1$ e $P_{t^{*}}(\cdot \mid F)=P_{t}(\cdot \mid F)$ (Loschi, Iglesias e Wechsler (1998)). Ou seja, a previsão de $E$ em $t^{*}$ é a previsão em $t$ dada a ocorrência do evento $F$, ou equivalentemente, no instante $t^{*}$ é certa a ocorrência de $F$ e a previsão de $E$ dado $F$ em $t$ é igual à previsão de $E$ feita no instante $t^{*}$ quando for certa a ocorrência de $F$.

Apesar de ser o procedimento mais utilizado em inferência bayesiana, o Condicionamento Bayesiano nem sempre fornece uma atualização para previsões ao longo do tempo. Outros procedimentos com o mesmo objetivo são o supercondicionamento e a regra geral de condicionamento (Loschi, Iglesias e Wechsler (1998)).

Uma das situações que exige algum procedimento distinto do Condicionamento Bayesiano é a ocorrência de um evento $F$ que não era sequer considerado no instante $t$ (percebe-se a diferença da situação em que $P(F)$ era zero). Aliás, a consideração de todos os eventos que possam ocorrer é humanamente impossível, e lidando-se com uma quantidade finita de eventos é muito natural a ocorrência de eventos inesperados.

Um fato a ser considerado é que a mudança de instante de tempo pode ocasionar uma mudança de opinião sobre $E$ sem que haja uma regra de atualização de $P_{t}(E)$ para $P_{t^{*}}(E)$. Conseqüentemente, a obtenção de distribuições de probabilidade ao longo do tempo não ocorre necessariamente conforme uma regra axiomática dependente de $P_{t}(E)$ e de algum 
fator de atualização, qualquer regra proposta para atualização é ad-hoc.

A propósito, convém ressaltar o aspecto positivo desta flexibilidade para propor previsões para diferentes instantes de tempo, já que, somente devido à ausência de regras rígidas de atualização, podem ser propostas previsões para situações totalmente inesperadas até um determinado momento.

Outra importante questão diz respeito à coerência coletiva. A construção de uma previsão como distribuição de probabilidades é intrinsecamente subjetiva, e conseqüentemente individual. Quando muitas previsões $P_{i}(E)$ para um mesmo evento $E$ em um dado instante de tempo estão disponíveis, não está definido um procedimento único para combinação destas previsões resultando em uma distribuição de probabilidades no sentido matemático. Com base no conceito subjetivista de probabilidade, teoricamente esta combinação não faz sentido, pois não seria a probabilidade subjetiva de um só indivíduo. Entretanto, na prática são comuns situações em que se pode combinar as opiniões de vários indivíduos de um grupo ou as previsões de diferentes sistemas de previsão (Capítulo 3) a fim de produzir uma única opinião ou previsão.

Devido à falta de uma definição de coerência coletiva nos moldes da coerência estática, são propostos diversos procedimentos para a construção de uma distribuição consenso (Loschi (1992)). Uma distribuição consenso é uma distribuição de probabilidades formada a partir de diversas funções de distribuição individuais. Tais procedimentos podem ser utilizados na escolha de um modelo de previsão quando dispomos de vários sistemas de previsão.

Um exemplo de distribuição consenso é o agrupamento linear de probabilidades em que a opinião sobre $\theta$ é da forma:

$$
P\left(Y_{n+1}\right)=\sum_{i=1}^{n} \omega_{i} P_{i}\left(Y_{n+1}\right),
$$

em que $P_{i}\left(Y_{n+1}\right)$ corresponde à opinião do i-ésimo indivíduo sobre a variável $Y_{n+1}, \omega_{i}>$ $0, \forall i=1, \ldots, n$ e $\sum_{i=1}^{n} \omega_{i}=1$.

$\mathrm{Na}$ ausência de uma definição de coerência coletiva, não existe regra obrigatória para a determinação dos pesos $\omega_{i}$ atribuídos à opinião de cada membro do grupo. Em Loschi 
(1992) são apresentados este e outros tipos de agrupamentos e suas propriedades. Um exemplo de propriedade satisfeita para o agrupamento linear de probabilidades é a preservação da unanimidade, ou seja, se todos os indivíduos do grupo consideram $P_{i}\left(Y_{n+1}\right)=\alpha$, então $P\left(Y_{n+1}\right)=\alpha$. 


\section{Capítulo 3}

\section{Sistemas de Previsão}

Em geral, os problemas de inferência estatística envolvem quantidades desconhecidas e para a obtenção de alguma informação sobre esta quantidade é utilizada uma amostra de dados. Na análise preqüencial, esta amostra é analisada de forma seqüencial, ou seja, com as $n$ primeiras observações é proposta uma previsão para a próxima. Deste modo, qualquer problema de inferência estatística pode ser analisado sob a ótica preqüencial.

Considere a situação em que ao longo do tempo são realizadas observações de uma variável de interesse, sendo $Y=\left(Y_{1}, Y_{2}, \ldots\right)$ a seqüência destas variáveis. Em cada instante $n, n=1,2, \ldots$, obtemos o valor observado $y_{n}$ da variável $Y_{n}$ e fornecemos uma previsão $P_{n+1}$ para a variável $Y_{n+1}$ até então desconhecida, e portanto, aleatória.

Note que neste ponto reside uma divergência fundamental entre estatísticos freqüentistas e bayesianos. Para freqüentistas, existem quantidades desconhecidas, porém "fixas", denominadas parâmetros, que podem assumir qualquer um dos diversos valores pertencentes ao espaço paramétrico. Sobre o espaço paramétrico não há medida de probabilidade que incorpore o conhecimento do pesquisador sobre o parâmetro. Entretanto, é importante salientar que na escola freqüentista surgem distribuições auxiliares de probabilidade, como são as distribuições amostrais, utilizadas, por exemplo, nas construções de intervalos de confiança.

Para bayesianos, qualquer quantidade desconhecida também pode variar em um determinado conjunto e, por seu caráter variável, ela será tratada como variável aleatória, 
podendo assumir qualquer valor no suporte de sua distribuição de probabilidades a priori.

Em conseqüência da adoção do axioma de coerência neste trabalho, uma previsão corresponde a uma distribuição de probabilidades, já que este é o único modo coerente de expressar incerteza sobre uma quantidade desconhecida num dado instante $n$.

Para a produção da previsão de $Y_{n+1}$, toda informação disponível até o instante $n$ deve ser utilizada, como, por exemplo, a seqüência de observações $y^{(n)}=\left(y_{1}, y_{2}, \ldots, y_{n}\right)$ do vetor $Y^{(n)}=\left(Y_{1}, Y_{2}, \ldots, Y_{n}\right)$; a seqüência de previsões $P_{n}$ para as observações já realizadas; covariáveis que forem julgadas relevantes para a previsão de $Y$ e opiniões do pesquisador relacionadas com o problema.

Será chamada de sistema de previsão qualquer método que forneça a cada instante $n$ uma previsão $P_{n+1}(\cdot)=P\left(\cdot \mid Y^{(n)}=y^{(n)}\right)$ para a próxima observação $Y_{n+1}$, embora esta previsão não seja necessariamente obtida como probabilidade condicional a partir de uma distribuição para o processo (apesar da notação utilizada). Esta previsão $P_{n+1}$ é uma função de probabilidades no caso discreto e uma densidade de probabilidades no caso contínuo.

Definição: Um Sistema de Previsão Probabilística (PFS) é qualquer algoritmo que forneça a previsão $P_{n+1}(\cdot)$ para cada valor de $n$ e vetor de observações $y^{(n)}$.

Exemplo: Seja $Y_{n}$ uma variável igual a 1 se ocorre um determinado evento E na n-ésima tentativa e 0 em caso contrário. Podemos propor um PFS em que $P_{n+1}(1)$ é igual à freqüência relativa de ocorrências de $\mathrm{E}$ até o instante $\mathrm{n}$.

Um PFS pode ser eventualmente definido pela distribuição condicional de $Y_{n+1}$ dado $Y^{(n)}=y^{(n)}$, a partir de uma distribuição de probabilidades conjunta associada ao processo $Y=\left(Y_{1}, Y_{2}, \ldots\right)$. Por outro lado, cada previsão pode ser entendida como uma probabilidade condicional, mas como os instantes de tempo em que as previsões foram produzidas são diferentes, a distribuição conjunta do processo não corresponde necessariamente ao produto destas previsões devido a ausência de definição de coerência temporal.

Em geral, diversos PFSs propostos não são obtidos como probabilidade condicional a partir de uma distribuição conjunta para o processo. Um claro exemplo de PFS não 
obtido deste modo é qualquer algoritmo que leva em conta o desempenho do sistema de previsão após $n$ observações na construção de uma previsão para $Y_{n+1}$.

De qualquer modo, um sistema de previsão probabilística (PFS) fornece, para cada instante $n$, uma previsão para a próxima observação $Y_{n+1}$, conhecidas as observações passadas $Y^{(n)}=y^{(n)}$ e outras informações disponíveis, e cada previsão consiste em uma distribuição de probabilidades. Assim sendo, todo PFS é coerente em cada instante $n$, ou seja, é estatisticamente coerente.

Tendo em vista a inexistência de uma definição de coerência temporal, um PFS pode fornecer previsões sem restrições quanto à construção da previsão $P_{n+1}$ conhecidas as observações e previsões anteriores. Particularmente, não há regra obrigatória para a construção de $P_{n+1}$, utilizando-se $P_{n}$ e $y^{(n)}$, com exceção dos casos em que outros julgamentos são feitos, como por exemplo o de permutabilidade, quando o Condicionamento Bayesiano é utilizado.

Também com o intuito de produzir previsões, considere uma família $\mathcal{P}=\left\{P_{\theta}, \theta \in \Theta\right\}$ de distribuições indexadas pelo parâmetro $\theta$. Uma previsão $P_{n+1, \theta}$, dependente de $\theta$, pode então ser produzida para $Y_{n+1}$. A utilização de um modelo paramétrico pode ser justificada, por exemplo, devido a julgamentos de invariância sobre quantidades observáveis no caso bayesiano, e pela adoção de modelos com parâmetros que "quantificam efeitos de variáveis explicativas" no comportamento da variável de interesse no caso freqüentista.

Adotando-se o ponto de vista subjetivista, a utilização dos parâmetros se deve a julgamentos de permutabilidade que resultam na expressão da distribuição conjunta para os dados observados como mistura de modelos paramétricos. Este tipo de resultado é demonstrado para o caso de variáveis dicotômicas permutáveis estendíveis no teorema da representação (de Finetti (1937)). Neste caso, a distribuição conjunta da amostra $y^{(n)}=\left\{y_{1}, \ldots y_{n}\right\}$ é obtida como alguma mistura de ensaios de Bernoulli com parâmetro $p$ :

$$
P\left(Y_{1}=y_{1}, \ldots, Y_{n}=y_{n}\right)=\int_{0}^{1} p^{\sum y_{i}}(1-p)^{n-\sum y_{i}} d F(p), \forall n=1,2, \ldots, \forall y_{i} \in\{0,1\}
$$


em que p é a variável aleatória correspondente ao limite da freqüência relativa

$$
\lim _{n \rightarrow \infty} \frac{\sum y_{i}}{n}
$$

e $F(p)$ é a função distribuição deste limite.

Este teorema, em seu caso mais geral, garante a possibilidade de escrevermos probabilidades conjuntas como misturas de verossimilhanças, como veremos a seguir.

Teorema da Representação (Caso Geral). Seja $\mathbf{Y}=\left(Y_{1}, Y_{2}, \ldots\right)$ um processo estocástico com valores em $\mathcal{X}^{\infty}$ com distribuição P. Então,

$\mathrm{P}$ permutável $\Leftrightarrow$ Existe única medida de probabilidade $\mu$ no conjunto $\mathcal{G}_{\mathcal{X}}$ das funções de distribuição sobre $\mathcal{X}$ tal que $\forall n \geq 1$ e $\forall y_{i} \in \mathcal{X}$ :

$$
\mathbf{P}\left(Y_{1} \leq y_{1}, \ldots, Y_{n} \leq y_{n}\right)=\int_{\mathcal{G}_{X}} \prod_{i=1}^{n} G\left(y_{i}\right) d \mu(G) .
$$

Este teorema garante a obtenção da probabilidade conjunta como mistura (produto) de probabilidades condicionais (dada a $G$ ). Aliando este resultado a outros julgamentos sobre as quantidades observáveis $Y_{i}$, como por exemplo uniformidade dado o valor de uma estatística suficiente, podemos obter a representação paramétrica para o processo $\mathbf{Y}$ (Zuazola (1993) e Wechsler (1993)).

De qualquer modo, a introdução de parâmetros em modelos de previsão não decorre da existência real de certas quantidades numéricas às quais denominamos parâmetros, e sim da maior facilidade de expressar a distribuição preditiva em função deles. Seguindo a mesma postura expressa na célebre afirmação de que "Probabilidade não existe" (De Finetti), o interesse da análise preqüencial se atém a quantidades que existam concretamente e possam ser observadas e mensuradas, ao contrário de parâmetros e "probabilidades" de ocorrência de algum evento.

Definição: Um Sistema de Previsão Estatística (SFS) consiste em uma técnica que utiliza uma família paramétrica de distribuições $\mathcal{P}=\left\{P_{\theta}, \theta \in \Theta\right\}$ para produzir um valor de $P_{n+1}$ para todo $n$, ou seja, é um PFS que utiliza alguma família $\mathcal{P}$ na construção de suas previsões. 
Utilizando-se $\mathcal{P}$ e os dados já disponíveis, inclusive $y^{(n)}$, uma previsão $P_{n+1, \theta}(\cdot)=$ $P_{n+1}\left(\cdot \mid \theta, y^{(n)}\right)$ dependente de $\theta$ pode ser proposta para $Y_{n+1}$. Com o intuito de eliminar a dependência de $\theta$ e fornecer uma previsão, podem ser propostos SFSs como o "plug-in" e o Bayesiano, descritos a seguir.

Um SFS do tipo "plug-in" consiste na construção de uma previsão $P_{n+1, \theta}$ para $Y_{n+1}$ e obtenção de $P_{n+1}$ por meio da substituição de $\theta$ em $P_{n+1, \theta}$ por alguma estimativa de $\theta$. A princípio, não há restrições quanto ao método de estimação de $\theta$ utilizado. Por exemplo, $P_{n+1, \theta}\left(y_{n+1}\right)=f\left(y_{n+1} \mid \theta\right)$ e $P_{n+1}\left(y_{n+1}\right)=f\left(y_{n+1} \mid \hat{\theta}\right)$.

O SFS Bayesiano atribui uma distribuição a priori para $\theta$ e determina o valor da previsão como sendo a distribuição preditiva:

$$
P_{n+1}(\cdot)=\int P_{n+1, \theta}(\cdot) d F_{n}(\theta)
$$

em que $F_{n}(\theta)$ é a distribuição a posteriori de $\theta$, dado $y^{(n)}$.

Exemplo: Sejam $Y_{n} \sim \mathcal{N}(\theta, 1), n=1,2, \ldots$, variáveis normais condicionalmente independentes, dado $\theta$. A previsão de $Y_{n+1}$ utilizando-se o método "plug-in" com estimação de máxima verossimilhança é dada por $Y_{n+1} \sim \mathcal{N}\left(\bar{y}_{n}, 1\right)$. Adotando-se uma distribuição a priori para $\theta$ igual a $\mathcal{N}(3,1)$, a distribuição preditiva é $\mathcal{N}\left(\frac{3+\sum_{i=1}^{n} y_{i}}{n+1}, \frac{n+2}{n+1}\right)$.

É importante ressaltar que a análise preqüencial não se aplica somente a problemas que tenham como objetivo principal a produção de previsões, estas entendidas como distribuições de probabilidade ou como resumos obtidos a partir destas distribuições, tais como médias, variâncias e intervalos de probabilidade. A análise preqüencial também pode ser utilizada para avaliar a adequabilidade de qualquer procedimento estatístico, seguindo a idéia de que a qualidade de um procedimento estatístico está relacionada ao desempenho do sistema de previsão a ele associado e de acordo com o pressuposto de que qualquer modelo estatístico pode ser utilizado na construção de um PFS (geralmente um SFS, no caso de modelos paramétricos). 


\section{Capítulo 4}

\section{Avaliação do sistema de previsão}

É desejável que um PFS apresente previsões bem próximas aos valores posteriormente observados. Para avaliar as discrepâncias entre as previsões e observações, as técnicas que medem a qualidade de um PFS devem depender somente das previsões geradas e correspondentes observações. Técnicas que apresentam esta característica seguem o Princípio Preqüencial Fraco (Dawid (1984)).

À semelhança do Princípio da Verossimilhança, esse princípio determina que a avaliação de um método não deve depender somente de previsões que poderiam ter sido feitas mas não foram.

Um bom exemplo de método de aferição de qualidade que não respeita o Princípio Preqüencial é apresentado a seguir.

Exemplo 1: Nível de significância (Dawid (1999)). Seja P uma distribuição para todo o processo $\left(Y_{n}\right)_{n}$, T uma estatística de teste e t o valor observado desta estatística tal que o nível descritivo observado $\hat{\alpha}=\mathrm{P}(T \geq t)$ quantifique a concordância entre as previsões e as observações. A avaliação das previsões a partir do valor de t não viola o princípio preqüencial, ao contrário do que ocorre com $\hat{\alpha}$, que depende da distribuição $\mathbf{P}$ e não somente das previsões para $Y_{n}$ e de seus valores observados.

Exemplo 2: Seja $P_{n}$ um PFS para a seqüência $Y_{n}$ com valores observados $y_{n}$. Considere 
a seqüência de transformadas integrais de probabilidade $u_{n}=P_{n}\left(Y_{n}<y_{n}\right)$. Métodos de avaliação do PFS baseados na seqüência $u_{n}$ respeitam o princípio preqüencial, já que cada $u_{n}$ depende somente da previsão $P_{n}$ e observação $y_{n}$.

Vale ressaltar que a avaliação de modelos estatísticos proposta na análise preqüencial é semelhante à técnica de validação cruzada (Stone (1974)). Nesta técnica, a previsão é obtida utilizando-se todos os dados disponíveis exceto a observação correspondente à previsão. Finalmente, são comparadas as observações excluídas e suas correspondentes previsões. A respeito da relação entre validação cruzada e análise preqüencial, Dawid (1997) comenta que, "como nesta última a previsão é obtida com base nas observações anteriores, não há problemas quanto ao aumento na quantidade de dados, são eliminados problemas de dependência entre avaliações para observações diferentes, simplificando a análise e a interpretação e permite o desenvolvimento de uma teoria mais geral e poderosa". Aliás, verificaremos mais adiante que inúmeros resultados assintóticos foram obtidos graças ao caráter seqüencial da análise.

\subsection{Tipos de medidas de avaliação}

Existem diversas medidas de avaliação para previsões sendo que estas podem ser divididas em medidas de confiabilidade e acurácia segundo Murphy e Winkler (1977). De um modo geral, são medidas que avaliam a discrepância entre observações e previsões e espera-se que um bom PFS minimize tais medidas.

A confiabilidade diz respeito à correspondência entre as previsões e as freqüências relativas observadas. A curva de calibração empírica é um exemplo de método que avalia a confiabilidade das previsões.

Uma outra característica a ser avaliada é a acurácia, que mede a diferença entre a previsão e sua respectiva observação. Medidas como o escore de Brier e a medida de De Finetti são medidas de acurácia.

Muitas vezes, medidas diferentes de avaliação avaliam aspectos bastante distintos das previsões. Por exemplo, considere uma variável dicotômica que pode assumir valores em 
$\{0,1\}$ (fracasso e sucesso, respectivamente) e os instantes $i_{1}, i_{2}, \ldots, i_{m}$ que apresentam previsões de sucesso iguais a 0,7 segundo algum PFS. Utilizando-se a medida escalar de calibração, este PFS será considerado bom se a freqüência relativa de sucessos nos instantes $i_{1}, i_{2}, \ldots, i_{m}$ for igual a 0,7 . Por outro lado, utilizando-se uma medida de acurácia, que mede a diferença entre a observação e a previsão, este PFS será julgado bom se as observações nos instantes $i_{1}, i_{2}, \ldots, i_{m}$ forem todas iguais a sucesso.

É importante ressaltar que o valor absoluto destas medidas não é por si só informativo ao ponto de indicar se um sistema de previsões é confiável ou acurado. Tanto as medidas de confiabilidade, quanto as de acurácia, são medidas cujos valores podem ser comparados para vários PFSs a fim de permitir a escolha de um único método de previsão.

A propósito, Murphy e Winkler (1977) apresentam o conceito de acurácia relativa (skill) que compara a acurácia de um sistema de previsão com a de outro método padrão de previsão.

A partir da distribuição de probabilidades em que consiste cada previsão, algumas medidas podem ser calculadas tais como a média e intervalos de probabilidade. Baseadas nessas medidas, outras avaliações para previsões podem ser feitas comparando-se:

- a média das previsões com as médias das observações;

- o valor p e a freqüência relativa da ocorrência de eventos pertencentes a uma região de probabilidade $\mathrm{p}$.

Para justificar a utilização de uma medida de avaliação de previsões é necessário conhecer seu comportamento para um bom sistema de previsões.

\subsection{Princípio Forte Preqüencial}

Seja $P^{*}$ a lei do processo $\left(Y_{n}\right)$ e considere a situação em que as observações do processo são geradas da distribuição $P^{*}$ e que as previsões $P_{n}$ também são obtidas a partir de $P^{*}$. Neste caso, a previsão seria a melhor possível já que quantifica exatamente a lei do processo gerador das observações. 
Em cada instante $n$, a existência da lei $P^{*}$, entendida como probabilidade subjetiva para o processo, é sempre garantida, apesar de sua especificação ser em geral inviável.

Conseqüentemente, o comportamento de um PFS pode ser analisado supondo-se a existência de uma distribuição de probabilidades (subjetiva) $P^{*}$ para o processo $\left(Y_{n}\right)$ e estudando-se o comportamento das medidas de avaliação do PFS se as previsões $P_{n}$ fossem obtidas a partir da distribuição $P^{*}$ subjacente ao processo.

Se o comportamento assintótico de um critério de avaliação para PFS independe da especificação de $P^{*}$, então tal critério segue o Princípio Forte Preqüencial (Dawid (1999)). Note que critérios deste tipo são totalmente robustos com relação a $P^{*}$, não necessitando sequer de sua especificação.

O comportamento esperado de medidas de avaliação de sistemas de previsão será discutido a seguir, sobretudo no que diz respeito à calibração, para a qual apresentamos detalhadamente várias razões para a expectativa de uma boa calibração.

\subsection{Calibração}

Considere um experimento com $Y_{n}$ sendo variável indicadora da ocorrência do evento $\mathrm{E}$ (sucesso) no instante $\mathrm{n}$ e $p_{n}$ sua respectiva previsão. Seja $w=\left(w_{1}, w_{2}, \ldots, w_{m}\right)$ uma sequência ordenada de valores pertencẹtes ao intervalo $(0,1)$ e $\delta=\left(\delta_{1}, \delta_{2}, \ldots \delta_{m}\right)$ tal que a união disjunta dos intervalos $I_{i}=\left[w_{i}-\delta_{i}, w_{i}+\delta_{i}\right]$ seja igual ao intervalo $[0,1]$. Dentre as $\eta_{i}$ observações cuja previsão pertence ao intervalo $I_{i}$, calculamos a proporção $f_{i}$ de sucessos. A curva de calibração empírica (ou curva de confiabilidade) é um gráfico de dispersão entre os valores $w_{i}$ e $f_{i}$.

Por exemplo, se 30 observações apresentam previsão no intervalo $[0,0 ; 0,2]$ (com $w_{1}$ $=0,1$ e $\left.\delta_{1}=0,1\right)$ e a proporção de sucessos nestas observações é 0,15 , o ponto $w_{1}=0,1$ e $f_{1}=0,15$ é plotado na curva de calibração. 


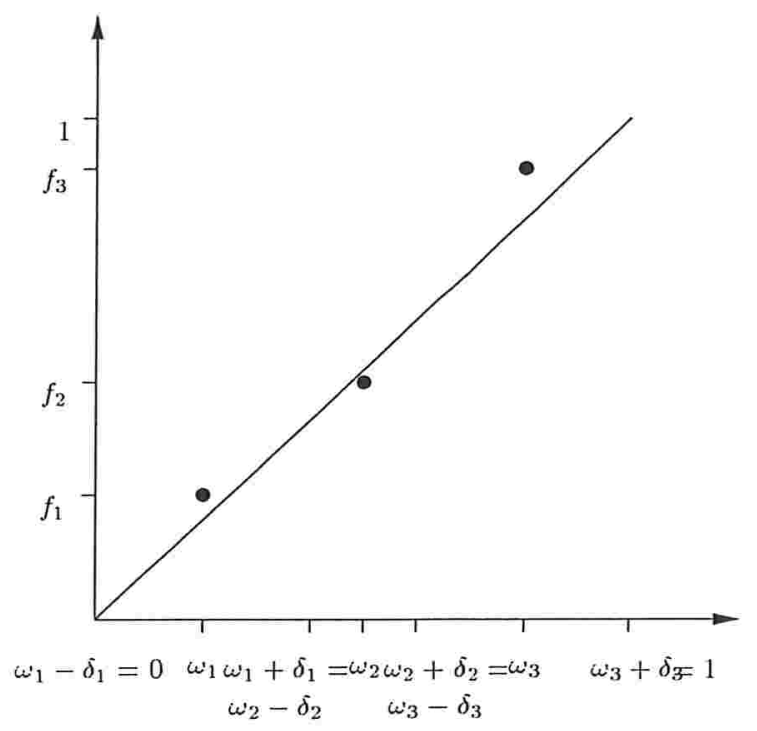

Fig. 1. Exemplo de gráfico de calibração com $m=3$.

O problema da escolha dos valores de $\mathrm{m}, w_{i}$ e $\delta_{i}$ é semelhante à escolha dos intervalos utilizados na construção de um histograma. Cada intervalo não pode ser nem muito pequeno nem muito grande, de modo que o número de previsões $\eta_{i}$ em cada intervalo seja razoável.

A curva de calibração empírica compara os valores $w_{i}$ e $f_{i}$ e um sistema de previsões perfeitamente calibrado. apresenta $w_{i}=f_{i}, \forall i=1, \ldots, m$. Na prática, esperamos encontrar $f_{i}$ bem próximo de $w_{i}$, para todo $i=1, \ldots, m$.

Uma curva de calibração também pode ser proposta para variáveis aleatórias discretas que assumam mais que dois valores. Para isso, devem ser consideradas as probabilidades da variável assumir cada categoria e as freqüências relativas das ocorrências de cada categoria. A ocorrência de cada categoria da variável é considerada como um evento dicotômico (ocorreu ou não). Analogamente, a curva de calibração é o diagrama de dispersão entre as probabilidades e as freqüências relativas da ocorrência de cada evento.

Outra generalização da curva de calibração para variáveis contínuas pode ser obtida considerando-se o evento ( $Y_{n}$ pertence ao intervalo de probabilidade $\mathrm{p}$ ), sendo este intervalo construído a partir da distribuição de probabilidades $P_{n}$ (previsão) para $Y_{n}$. A 
freqüência relativa $f$ de ocorrência do evento $\left(Y_{n}\right.$ pertence ao intervalo) deve ser próxima de $p$. Este procedimento pode ser repetido para diferentes valores de $p$ e para vetores aleatórios construindo-se regiões de probabilidade.

Em geral, para os mais variados tipos de variáveis aleatórias são propostas variáveis dicotômicas que assumem valor 1 ou 0 , caso um determinado evento - função destas variáveis - ocorra ou não. Assim, sua probabilidade obtida a partir da previsão para a variável aleatória será comparada com a proporção de sua ocorrência.

Quando uma curva mal calibrada é obtida, a informação dada pela curva pode ser utilizada para corrigir as próximas previsões a fim de fornecer um sistema melhor calibrado. Por exemplo, se sempre que a previsão está em torno de 0,1 a proporção de sucessos é 0,3 , então as previsões estão subestimadas e pode-se apresentar previsão igual a 0,3 sempre que o sistema fornece previsão 0,1 . Este tipo de procedimento é chamado de recalibração e sua relação com a noção de coerência será discutida mais adiante (Capítulo 6).

Por que é esperado que um bom PFS seja bem calibrado?

Um sistema de previsão com alta confiabilidade apresenta previsões com valores bem próximos das freqüências relativas. Uma justificativa assintótica para este comportamento é dada supondo-se a existência da distribuição subjacente ao processo $P^{*}$ e com as previsões para a variável binária $Y_{n}$ dadas por

$$
P_{n}=\mathbb{E}_{P^{*}}\left(Y_{n} \mid Y_{1}, \ldots, Y_{n-1}\right)
$$

Pela lei forte dos grandes números para martingais (Dawid $(1982,1999)$ e Stout $(1974)$ Teorema 3.3.1), tem-se que com probabilidade $P^{*}$ igual a 1

$$
\lim _{N \rightarrow \infty} \frac{1}{N} \sum_{n=1}^{N}\left(Y_{n}-P_{n}\right)=0 .
$$

Este comportamento assintótico independente da especificação de $P^{*}$ segue o Princípio Forte Preqüencial e justifica o uso da curva de calibração. Assim, para um bom PFS, ou seja, para um sistema de previsões que capta a lei do processo gerador das observações, as freqüências relativas devem estar bem próximas das previsões. 
A validade deste resultado para subseqüências justifica a construção de uma mesma curva de calibração para diversos eventos. Neste caso, também espera-se que para um bom PFS as freqüências relativas e previsões estejam muito próximas.

Para os adeptos da escola freqüentista de probabilidade, um bom sistema de previsão deve apresentar alta confiabilidade para um grande conjunto de dados e previsões, já que, segundo sua teoria, probabilidade é justamente o limite da freqüência relativa. Assim, se $p$ é a probabilidade de ocorrência de um evento, então a freqüência relativa de ocorrência deste evento em um número infinito de repetições de um experimento realizadas independentemente e sob as mesmas condições experimentais é $p$. A definição freqüentista de probabilidade apresenta uma grande limitação por estar associada à independência, que por sua vez está definida a partir do conceito de probabilidade.

Outra característica da curva de calibração é que nela podem ser avaliadas as ocorrências de eventos bastante diferentes. Um exemplo de curva de calibração que envolve eventos diferentes em uma mesma análise pode ser encontrada em Arruda (2000), onde são apresentadas as probabilidades e freqüências relativas de vitória, empate e derrota de diversos times em jogos de futebol num mesmo gráfico. Seguindo a definição freqüentista de probabilidade, o limite da freqüência relativa para os eventos com previsão $p$ não é necessariamente igual a $p$ conforme a lei dos grandes números, a menos que sejam válidas hipóteses como a de independência, o que não ocorre para os três resultados de um jogo de futebol.

Mesmo a partir da definição freqüentista de probabilidade, é possível estudar o comportamento esperado da curva de calibração. Supondo-se a existência de uma lei $P^{*}$ para o processo todo $\left(Y_{n}\right)_{n}$, ou seja, que as observações são geradas segundo esta distribuição de probabilidades, obtemos que o PFS $P$ é igual à lei do processo $P^{*}$ se e somente se o limite da freqüência relativa é igual a $P$, ou seja, ocorre calibração perfeita, e isto independe da especificação de $P^{*}$. Assim, o uso da curva de calibração para avaliação de previsões segue o princípio forte preqüencial.

Seguindo a visão subjetivista, uma distribuição de probabilidades para o processo é a. opinião de um determinado indivíduo sobre este processo, o que garante sua existência. 
A situação em que cada previsão $P_{n}$ para $Y_{n}$ dadas as informações disponíveis até o instante $n$ é igual à probabilidade condicional obtida a partir da lei do processo $\left(P^{*}\right)$ corresponde à situação de melhor previsão possível, pela própria definição de previsão como probabilidade.

Se, conforme a lei $P^{*}$ do processo, as previsões em alguns instantes são iguais a $p$, a partir de julgamentos de permutabilidade para estas observações obtém-se a convergência do limite da freqüência relativa para uma variável aleatória com média $p$.

\subsection{Medida escalar de confiabilidade e Escore de Brier}

Considere o processo estocástico $\left(Y_{n}\right)$ em que cada variável aleatória $Y_{n}$ pode assumir $r$ valores. Para as variáveis observadas $y_{n}$, defina o vetor $\mathrm{r}$-dimensional $\mathrm{y}_{n}$ tal que cada componente $y_{n j}=\mathbf{1}_{\left(y_{n}=j\right)}$, com 1 denotando a função indicadora. Seja $\mathbf{p}_{n}$ uma previsão vetorial para a variável aleatória $Y_{n}$ com cada componente $p_{n j}$ igual à probabilidade do evento $\left(Y_{n}=j\right)$ e $\mathbf{P}=\left\{\mathbf{p}^{i}, \quad i=1, \ldots, m\right\}$ o conjunto dos diferentes valores assumidos pelos $N$ vetores de previsões.

Uma medida de confiabilidade que mede a distância entre a previsão e a freqüência relativa pode ser expressa por

$$
M c=\frac{1}{N} \sum_{i=1}^{m} N_{i}\left(\mathrm{p}^{i}-\mathrm{f}^{i}\right)\left(\mathrm{p}^{i}-\mathrm{f}^{i}\right)^{\prime},
$$

em que $N_{i}$ é o número de vezes que a previsão assume o valor $\mathrm{p}^{i}, N=\sum_{i=1}^{m} N_{i}$ e $\mathrm{f}^{i}=$ $N_{i}^{-1} \sum_{n=1}^{N} \mathbf{y}_{n} \mathbf{1}_{\left(\mathrm{p}_{n}=\mathrm{p}^{i}\right)}$ é o vetor de freqüências relativas de cada uma das r categorias com relação aos $N_{i}$ valores observados com previsão $\mathrm{p}^{i}$.

Esta medida pode ser utilizada como uma medida resumo da curva de calibração.

Uma outra medida utilizada neste tipo de situação é o escore de Brier (Brier (1950)), também conhecido como Probability Score (PS), e definido pela expressão 


$$
P S=\frac{1}{N} \sum_{n=1}^{N} \sum_{j=1}^{r}\left(p_{n j}-\mathbf{1}_{\left(y_{n}=j\right)}\right)^{2}=\frac{1}{N} \sum_{n=1}^{N}\left(\mathrm{p}_{n}-\mathrm{y}_{n}\right)\left(\mathrm{p}_{n}-\mathrm{y}_{n}\right)^{\prime} .
$$

O escore de Brier mede a distância entre a probabilidade atribuída a um evento e a ocorrência do mesmo.

Uma propriedade interessante do escore de Brier (desenvolvida por Sanders (1958) e discutida em Murphy e Epstein (1967) e Murphy e Winkler (1972)) é que ele pode ser escrito como soma da medida escalar de confiabilidade e uma medida de variabilidade das observações

$$
P S=\frac{1}{N} \sum_{i=1}^{m} N_{i}\left(\mathrm{p}^{i}-\mathrm{f}^{i}\right)\left(\mathrm{p}^{i}-\mathrm{f}^{i}\right)^{\prime}+\frac{1}{N} \sum_{i=1}^{m} N_{i} \mathrm{f}^{i}\left(\mathrm{u}-\mathrm{f}^{i}\right)^{\prime}
$$

em que u é um vetor $r \times 1$ e $u_{l}=1, \forall l=1 \ldots, r$.

Nesta última expressão podemos identificar a primeira parcela como sendo a medida escalar de confiabilidade (4.1) e a segunda parcela $V=\frac{1}{N} \sum_{i=1}^{m} N_{i} \mathbf{f}^{i}\left(\mathbf{u}-\mathrm{f}^{i}\right)^{\prime}$ referente à variabilidade do processo está associada ao que se chama de resolução do previsor.

Deste modo, a utilização do escore de Brier para aferição da qualidade das previsões é equivalente à da medida de confiabilidade com o acréscimo de um valor que corresponde à variabilidade do processo, que é intrínseco ao processo e não diz respeito à qualidade da previsão.

\subsection{Transformada da integral de probabilidade}

Esta técnica baseia-se no fato da função distribuição de uma variável aleatória ter distribuição uniforme em [0,1] (Esteves(1997), por exemplo). Assim, para a seqüência de previsões $P_{n}$ para $Y_{n}$ e valores observados $y_{n}$ é esperado, sob a distribuição $P^{*}$ subjacente ao processo, que os valores de $u_{n}=P_{n}\left(Y_{n} \leq y_{n}\right)$ sejam independentes e apresentem distribuição empírica aproximadamente uniforme. Vários testes podem ser utilizados para verificarmos se $u_{n}$ tem distribuição uniforme. Note que critérios que se baseiam nos 
valores de $u_{n}$ respeitam o Princípio Preqüencial, pois dependem somente das previsões $P_{n}$ e das observações $y_{n}$.

Esta técnica também permite a atualização das previsões se verificado algum desvio da função de distribuição empírica com relação à uniforme. Para isso, considere $G_{n}$ um bom PFS construído para prever os valores de $u_{n}$. Assim, $G_{n}$ pode ser utilizado para recalibrar o sistema de previsões $P_{n}$, sendo $G_{n}\left(P_{n}(\cdot)\right)$ a nova função distribuição para a variável de interesse $Y_{n}$.

\subsection{Funções de perda}

A escolha de um sistema de previsão pode ser entendido como um problema de teoria da decisão em que a ação a ser tomada em cada instante $n$ é a escolha de uma previsão $P_{n+1}$ para a variável $Y_{n+1}$ (Dawid (1992)).

Seja $L_{n}(y, p)$ uma função de perda quando $Y_{n}=y$ e a previsão para $Y_{n}$ é $P_{n}=p$. Um PFS $P$ pode ter seu desempenho avaliado pela função de perda preqüencial total

$$
L_{N}^{*}(P)=\sum_{n=1}^{N} L_{n}\left(y_{n}, P_{n}\right) .
$$

Esta função de perda leva em conta a perda de todas as previsões já enunciadas até o instante $\mathrm{N}$.

Do mesmo modo, um sistema de previsão P definido como um SFS pode ser escolhido numa família $\mathcal{P}=\left\{P_{\theta}, \theta \in \Theta\right\}$, em que cada previsão $P_{n}(\theta)$ depende de $\theta$. Um modo "plug-in" de obtermos uma previsão independente de $\theta$ é definido pela substituição de $\theta$ pelo valor $\hat{\theta}$ que minimiza a função de perda

$$
L_{N}^{*}(\theta)=\sum_{n=1}^{N} L_{n}\left(y_{n}, P_{n}(\theta)\right) .
$$

Para avaliarmos as previsões produzidas pelo SFS acima, definimos a função de perda preqüencial acumulada por

$$
L_{N}^{*}(P)=\sum_{n=1}^{N} L_{n}\left(y_{n}, P_{n}(\hat{\theta})\right) .
$$


Como já foi estudado anteriormente, a eliminação do parâmetro $\theta$ também pode ser feita de modo bayesiano e a avaliação segue em função de $L_{N}^{*}(P)$.

Uma característica desejável em um PFS pode ser a minimização de alguma função de perda definida, sendo que cada função de perda estão associada a diferentes propriedades de interesse para as previsões.

Podemos escolher procedimentos que utilizam funções de perda $L_{n}$ tais que o mínimo da $\mathbb{E}_{Q_{n}}\left[L_{n}\left(Y_{n}, P_{n}\right)\right]$ é obtido quando $P_{n}=Q_{n}$, ou seja, elas garantem que a perda esperada é mínima quando a previsão (opinião) $P_{n}$ é sincera (Dawid (1986)). Tais funções de perda são chamadas de regras próprias de escore e dizemos que são estritas se este mínimo é único. Regras deste tipo podem ser utilizadas para incentivar um indivíduo a enunciar uma previsão $P_{n}$ sincera, já que, se ele acha que a distribuição de $Y_{n}$ é $Q_{n}$, então a previsão $P_{n}=Q_{n}$ minimiza a função de perda esperada. Neste contexto, sistemas de previsão que minimizam uma regra própria apresentam previsão mais próxima da distribuição $Q_{n}$ de $Y_{n}$.

Exemplos de regras próprias de escore são o escore de Brier e o escore logaritmo. O módulo das diferenças entre previsões e observações é um exemplo de regra imprópria. Estas regras de escore podem ser utilizadas na definição da função de perda preqüencial definida para as $N$ observações.

Para estudar o comportamento assintótico da função de perda preqüencial (4.2), considere a lei $P^{*}$ subjacente ao processo. Para $L_{n}$ uniformemente limitada e sob algumas suposições de mensurabilidade (Dawid e Vovk (1999) e Shiryayev (1984) corolário do teorema VII.5.1), a diferença

$$
L_{N}^{*}(P)-L_{N}^{*}(Q)
$$

é sob $P^{*}$ um supermartingal com incrementos limitados, e com probabilidade $P^{*}$ igual a 1 temos

$$
\liminf _{N \longrightarrow \infty}\left(L_{N}^{*}(P)-L_{N}^{*}(Q)\right)<\infty .
$$

Esta propriedade assintótica sugere a rejeição de sistemas de previsão $P$ com função de perda acumulada $L_{N}^{*}(P)$ se existe um sistema $Q$ tal que 


$$
\liminf _{N \rightarrow \infty}\left(L_{N}^{*}(P)-L_{N}^{*}(Q)\right)=\infty
$$

Na prática, estas funções de perda são medidas de aferição da qualidade de um sistema de previsão com um número finito de previsões, e este comportamento assintótico nos sugere a escolha de um sistema que minimize a função de perda preqüencial acumulada.

\subsection{Escore de Brier e medida de De Finetti}

Considere o processo estocástico $\left(Y_{n}\right)$ em que cada variável aleatória discreta $Y_{n}$ pode assumir $r$ valores. Para as observações $y_{n}$, defina o vetor $\mathbf{r}$-dimensional $\mathbf{y}_{n}$ tal que cada componente $y_{n j}=\mathbf{1}_{\left(y_{n}=j\right)}$, com 1 denotando a função indicadora. Seja $\mathrm{p}_{n}$ uma previsão vetorial para a variável aleatória $Y_{n}$ com cada componente $p_{n j}$ igual à probabilidade do evento $\left(Y_{n}=j\right)$ e $\mathbf{P}=\left\{\mathbf{p}^{i}, \quad i=1, \ldots, m\right\}$ os diferentes valores assumidos pelos $N$ vetores de previsões.

Como visto anteriormente, a medida escalar de confiabilidade pode ser decomposta em duas parcelas, uma delas corresponde ao escore de Brier e a outra se refere à variabilidade do processo.

O escore de Brier (Brier (1950)), também conhecido como Probability Score, é uma função de perda própria que corresponde à diferença quadrática entre a probabilidade atribuída ao evento $\left(Y_{n}=j\right)$ e a ocorrência do mesmo. Este escore é expresso por

$$
P S=\frac{1}{N} \sum_{n=1}^{N} \sum_{j=1}^{r}\left(p_{n j}-\mathbf{1}_{\left(y_{n}=j\right)}\right)^{2}=\frac{1}{N} \sum_{n=1}^{N}\left(\mathrm{p}_{n}-\mathrm{y}_{n}\right)\left(\mathrm{p}_{n}-\mathrm{y}_{n}\right)^{\prime} .
$$

Seja $I^{r}$ o simplex contido em $\Re^{r}$ e definido pela equação $\sum_{i=1}^{r} x_{i}=1, \operatorname{com} x_{i} \geq 0, \forall i=$ $1, \ldots, r$. Associando cada observação $y_{i}$ a um vértice $\mathrm{y}_{i}$ do simplex $I^{r}$, e cada previsão a um ponto deste simplex, a medida de De Finetti (1972) corresponde à distância euclidiana entre a observação e sua respectiva previsão. Esta medida também é uma regra própria.

Para a n-ésima observação, a medida de De Finetti é definida por

$$
M f_{n}=\sqrt{\left(\mathrm{p}_{n}-\mathrm{y}_{n}\right)\left(\mathrm{p}_{n}-\mathrm{y}_{n}\right)^{\prime}} .
$$


Uma medida definida para $N$ observações é a média aritmética destas distâncias

$$
M f=\frac{1}{N} \sum_{n=1}^{N} \sqrt{\left(\mathrm{p}_{n}-\mathrm{y}_{n}\right)\left(\mathrm{p}_{n}-\mathrm{y}_{n}\right)^{\prime}} .
$$

Note que a medida de De Finetti é uma medida bastante parecida com o escore de Brier, sendo que esta última considera a distância euclidiana ao quadrado.

Analogamente a essas medidas podemos considerar a função de perda correspondente ao valor absoluto da diferença entre a observação e a previsão

$$
M a=\frac{1}{N} \sum_{n=1}^{N}\left|y_{n}-P_{n}\right|,
$$

em que $P_{n}$ é a probabilidade de $Y_{n}=y_{n}$.

Esta medida não é uma regra própria de escore. Por exemplo, considere $Y$ uma variável indicadora da ocorrência de cara em um lançamento de moeda. Se a probabilidade de $Y$ ser cara é $Q(1)=0,8$, então o mínimo do valor esperado $\mathbb{E}_{Q}[M a(Y, P)]$ é atingido quando $P(1)=1$.

\subsection{Verossimilhança Preqüencial}

Considere o processo estocástico $\left(Y_{n}\right)$ com cada variável aleatória $Y_{n}$ assumindo valores em um conjunto $\mathcal{X}$ qualquer. Seja $P_{n}$ a previsão para $Y_{n}$ dadas as observações e previsões anteriores.

A verossimilhança preqüencial $L_{P, N}$ para o sistema de previsões $P$ e seqüência de dados $y^{(N)}($ Dawid (1994)) é definida por

$$
L_{P, N}=\prod_{k=1}^{N} P_{n}\left(y_{n}\right),
$$

em que $P_{n}\left(y_{n}\right)$ é a probabilidade de $Y_{n}=y_{n}$ segundo a previsão $P_{n}$.

A partir da verossimilhança preqüencial, podemos obter a log-verossimilhança preqüencial e trabalhar com a função de perda acumulada

$$
L_{N}^{*}(P)=-\sum_{n=1}^{N} \log P_{n}\left(y_{n}\right) .
$$


Essa é a função de perda acumulada considerando-se o escore logaritmo - $\log \left(P_{n}\left(y_{n}\right)\right)$, que constitui uma regra própria. Para sistemas de previsão $P$ com valores de previsão $P_{n}$ limitados, valem todos os resultados assintóticos apresentados para funções de perda.

Para dois sistemas de previsão $P$ e $Q$, podemos considerar a razão entre as verossimilhanças preqüenciais

$$
\Lambda_{N}(Q, P)=\frac{L_{Q, N}}{L_{P, N}}
$$

Considerando $P$ como lei subjacente ao processo (medida de probabilidade subjetiva), $\Lambda_{N}(Q, P)$ é um martingal não negativo e converge a um valor finito $\Lambda$ com probabilidade 1. Deste modo, a observação de uma seqüência de razões de verossimilhanças com limite tendendo a infinito nos leva a rejeitar o sistema $P$. Invertendo-se os sistemas desta razão obtemos que razões tendendo a zero nos levam a rejeitar o sistema $Q$ (Dawid (1984)).

Quando $P$ é uma medida absolutamente contínua com respeito a $Q, P \ll Q, \Lambda$ é positivo com probabilidade 1 , tanto sob $P$, quanto $Q$. Assim, só poderia ser rejeitado o sistema $P$. O teorema de Blackwell e Dubins (1962) nos garante que com probabilidade $P$ igual a 1 as distribuições condicionais para o futuro obtidas a partir das leis $P$ e $Q$, dado $Y^{(n)}$, serão assintoticamente indistinguíveis. Assim, se $P$ e $Q$ são mutuamente absolutamente contínuas $(P \sim Q)$, podemos inverter o numerador e o denominador da razão de verossimilhanças e não sermos capazes de optar por um único sistema de previsão. Nesta situação, $P$ e $Q$ são denominados PFSs equivalentes.

\subsection{Consistência e Eficiência Preqüencial}

Considere a família de distribuições $\mathcal{P}=\left\{P_{\theta}, \theta \in \Theta\right\}$, com $\Theta \subseteq \mathbb{R}^{k}$ e seja $P^{*}$ um PFS tal que $P_{\theta} \ll P^{*}$, para todo $\theta \in \Theta$. Pelo teorema de Blackwell e Dubins (1962), com probabilidade igual a 1 sob qualquer $P_{\theta} \in \mathcal{P}$, as previsões para o futuro obtidas pela $P^{*}$ serão assintoticamente indistinguíveis das previsões feitas a partir do "correto" $P_{\theta}$. Neste caso, $P^{*}$ será chamado de completamente consistente para $\mathcal{P}$ (Dawid (1984)). Vale notar que a utilização da lei de Cromwell sugere a obtenção de um PFS completamente 
consistente, já que recomenda a escolha de $P^{*}$ que domine a família $\mathcal{P}$.

Neste contexto, como nem sempre podemos obter consistência completa, definimos consistência (simples) quando a previsão $P_{n}^{*}$ para a próxima observação é assintoticamente igual à previsão obtida por $P_{n, \theta}$ (Dawid (1984)). Por exemplo, se $\bar{X}_{n} \rightarrow \theta, P_{n}^{*}=\mathcal{N}\left(\bar{x}_{n}, 1\right)$ e $P_{n, \theta}=\mathcal{N}(\theta, 1)$, então $P_{n}^{*}$ e $P_{n, \theta}$ são assintoticamente iguais e $P_{n}^{*}$ será considerado um PFS consistente.

Seja R um SFS bayesiano com priori $\pi(\theta)>0, \forall \theta \in \Theta$ e Q um SFS arbitrário para $\mathcal{P}$. Então para a razão de verossimilhanças preqüenciais $\Lambda_{N}(Q, R)(4.3)$

$$
1=R\left(\Lambda_{N}(Q, R) \rightarrow \Lambda<\infty\right)=\int P_{\theta}\left(\Lambda_{N}(Q, R) \rightarrow \Lambda<\infty\right) \pi(\theta) d \theta
$$

e portanto, $P_{\theta}\left(\Lambda_{N}(Q, R) \rightarrow \Lambda<\infty\right)=1$ para quase todo $\theta$, o que implica que $\mathrm{R}$ não pode ser superado, exceto para um conjunto de parâmetros com medida nula (Dawid (1984)). Qualquer SFS R com esta propriedade é chamado de eficiente. Em geral, um SFS é eficiente quando sua previsão é mutuamente absolutamente contínua com respeito a um SFS bayesiano com priori positiva para todo $\theta \in \Theta$. 


\section{Capítulo 5}

\section{Considerações sobre a}

\section{implementação da Análise}

\section{Preqüencial}

A análise preqüencial consiste na análise de dados de forma seqüencial, obtendo-se sempre previsões para a próxima observação. Cada previsão corresponde a uma distribuição de probabilidades e pode ser obtida a partir de modelos probabilísticos para os dados (verossimilhança) e de modelos que incorporem o conhecimento do pesquisador sobre quantidades de interesse (priori). Finalmente, as observações são comparadas com suas respectivas previsões para avaliar o desempenho dos modelos envolvidos e das técnicas de previsão.

Em geral, a implementação da análise preqüencial compreende as seguintes etapas:

- Organização da seqüência de dados;

- Escolha do modelo probabilístico para os dados da amostra e para outras informações disponíveis;

- Escolha do método de previsão e obtenção da previsão;

- Avaliação do sistema de previsão. 
Apesar da ordenação destas etapas parecer bastante natural, em geral podemos propor diversos modelos e métodos de previsão. A escolha de um único sistema de previsão será baseada na avaliação de tais sistemas.

Nas seções seguintes são apresentados alguns aspectos referentes à escolha de modelos e de métodos de previsão.

\subsection{Escolha de modelos}

Uma das preocupações da Estatística é a adoção de um modelo estatístico a ser utilizado com diversos objetivos, não só preditivos, como para testes de hipóteses e estimação.

A análise preqüencial pode ser utilizada como ferramenta na escolha de um modelo que será utilizado posteriormente de acordo com os objetivos da análise. A busca por um modelo com bom desempenho preditivo é fundamental, já que na etapa de avaliação serão comparadas as previsões obtidas a partir do modelo a ser avaliado e quantidades efetivamente observadas.

A escolha do modelo pode ser baseada nas medidas de avaliação apresentadas, como a calibração e alguma função de perda preqüencial total. Outros critérios para esta escolha podem ser propostos levando-se em consideração o número de parâmetros dos modelos e medidas de desempenho preqüencial. Estes critérios visam a obtenção de modelos parcimoniosos.

Alguns critérios são propostos em função da verossimilhança preqüencial. Dawid (1997) comenta que a verossimilhança preqüencial pode ser aproximada pelo critério de Jeffreys-Schwartz (Smith (1988)) expresso por $\log \left(L_{x^{(n)}}(\hat{\theta})\right)-0,5 d \log (n)$, em que $d$ é a dimensão do parâmetro. Assim, a abordagem preqüencial já incorpora uma certa penalidade referente à complexidade dos modelos.

Um exemplo deste tipo de critério é proposto para a escolha da ordem de uma cadeia de Markov em Dorea et al. (1997). Neste caso, os critérios AIC, BIC e de mínima entropia visam a obtenção da ordem da cadeia que maximiza a verossimilhança sem um acréscimo muito grande no número de parâmetros. Veremos na seção 7.2 que o critério que maximiza 
a verossimilhança preqüencial neste caso não indica qual a ordem correta do processo.

Não obstante, em Dawid (1992b) um critério baseado na maximização da verossimilhança preqüencial é utilizado para a escolha da ordem do modelo autoregressivo. A partir de um conjunto de dados simulados, verificou-se que, em geral, este critério encontra a verdadeira ordem do modelo.

\subsection{Escolha do sistema de previsão}

A escolha de um método de previsão está diretamente relacionada ao seu desempenho preditivo, sendo este um aspecto primordial da análise preqüencial. Mesmo quando o objetivo da análise não for estritamente preditivo, a escolha de um bom modelo pode ser feita com base na sua capacidade de propor previsões bem próximas das observações. Para a avaliação desta capacidade é necessária a adoção de um sistema de previsão, e espera-se que este seja o melhor possível para cada modelo.

Apesar de termos definido sistemas de previsão com base nos valores já observados, todo conhecimento disponível até o instante $n$ deve ser incorporado ao modelo. Deste modo, podemos incluir, por exemplo, covariáveis e nossa própria opinião sobre a distribuição das variáveis em estudo.

A propósito, observe que no instante $n+1$ dispomos das seqüências de observações $y^{(n)}$ e de suas respectivas previsões $P_{1}, \ldots, P_{n}$. Portanto, podemos utilizar a informação proveniente da avaliação do sistema de previsões para produzir uma nova previsão, ou seja, recalibrar o sistema como foi mencionado no Capítulo 4.

Também a respeito da escolha do sistema de previsão, podemos considerar situações em que diversas previsões $P_{k, n}, k=1, \ldots, K$ são propostas para a mesma variável $Y_{n}$. Devido à inexistência de uma definição de coerência coletiva (seção 2.1), há inúmeros modos de propor uma só previsão (distribuição consenso) a partir de várias (Loschi (1992)).

Quanto à avaliação de um modelo estatístico, podemos construir, por exemplo, um sistema estatístico de previsão (SFS) utilizando este modelo para definir a família de distribuições $\mathcal{P}=\left\{P_{\theta}, \theta \in \Theta\right\}$. Após a construção de previsões pertencentes a $\mathcal{P}$, a 
eliminação da dependência de $\theta$ pode ser obtida pelos métodos "plug-in" ou bayesiano.

Para análises estatísticas cujo objetivo não é a produção de previsões, o modelo estatístico utilizado pode ser avaliado preqüencialmente. Assim, num teste de igualdade de duas médias utilizando-se o modelo normal, este modelo pode definir a família paramétrica $\mathcal{P}=\left\{P_{\theta}, \theta \in \Theta\right\}$. A escolha da técnica de previsão não fica definida pelo problema e diversas técnicas podem ser utilizadas. 


\section{Capítulo 6}

\section{Recalibração vs Coerência}

Dawid (1982) demonstra que com probabilidade $\Pi$ igual a 1 , se $\eta_{i} \rightarrow \infty$, então $f_{i}-\pi_{i} \rightarrow$ 0 , em que $\Pi$ é uma distribuição conjunta para todo o processo e dentre as $\eta_{i}$ observações cuja previsão pertence ao intervalo $I_{i}$, calculamos a proporção $f_{i}$ de sucessos e a média $\pi_{i}$ das previsões.

Isto quer dizer que assintoticamente o previsor tem certeza de que suas previsões são bem calibradas (Loschi(1992)) (este resultado vale com $\Pi$-probabilidade 1, se é que isto tem algum significado).

Para obter este resultado, Dawid exige somente que a inclusão dos eventos que compõem a curva de calibração seja determinada antes da ocorrência de tais eventos e utiliza uma distribuição conjunta $\Pi$ sobre todos os eventos obtidos a partir das futuras observações $\left(Y_{n}\right)$.

O teorema estabelece a boa calibração assintótica para todo conjunto de dados. Um conjunto de observações construído a partir da distribuição ПI que serve como contra exemplo para este resultado é apresentado em Oakes (1985). Assim, não há como garantir a existência de um sistema de previsão calibrado para qualquer conjunto de observações $\left(y_{n}\right)$, o que reforça a necessidade da contínua avaliação das previsões obtidas e eventuais recalibrações.

Um dilema entre recalibração e coerência é discutido em vários trabalhos (Dawid (1982) e Loschi (1992)). 
É amplamente discutida na literatura a incoerência da recalibração, ou seja, do procedimento que envolve a produção da previsão $p_{n}$ e posterior utilização de uma uma transformação $\gamma$ para recalibrar esta previsão, sendo que ao enunciar $p_{n}$ a informação disponível no momento de enunciar $p_{n}$ e $\gamma\left(p_{n}\right)$ é a mesma.

$\mathrm{Na}$ abordagem preqüencial, é esperado que sejam utilizadas todas as informações disponíveis até o presente, inclusive a própria curva de calibração empírica para a produção de previsões. Assim, uma previsão $p_{n+1}$ que ainda não utilizou a informação da curva de calibração pode ser entendida como uma previsão preliminar que ainda será recalibrada. Em nenhum momento estão sendo propostas duas previsões distintas para a mesma quantidade, o que seria incoerente.

É importante verificar que a recalibração é coerente temporalmente, já que isto requer somente a coerência estática da previsão em cada instante e a atualização da previsão pode ser obtida sem restrições quanto à transformação adotada. Lindley salienta que a informação de má calibração altera a informação até então disponível tornando, portanto, natural a recalibração (Loschi (1992), comentário de Dawid(1982)).

O próprio Dawid (discussão (1982)) ressalta a natureza dinâmica dos sistemas de previsão em que cada previsão é produzida num instante diferente e não deve ser entendida como uma probabilidade condicional calculada a partir de uma probabilidade conjunta $\Pi$ escolhida no instante zero para todos os possíveis eventos da seqüência $\left(Y_{n}\right)$. 


\section{Capítulo 7}

\section{Aplicações}

Com o intuito de ilustrar a implementação da análise preqüencial em problemas reais de inferência estatística, quatro aplicações são apresentadas e discutidas:

- Índice de mortalidade infantil

- Modelagem de contornos acentuais no português

- Sobrevivência após o diagnóstico de mieloma

- Séries temporais com modelos autoregressivos

\section{1 Índice de mortalidade infantil}

O desenvolvimento de estudos que proporcionem a previsão do risco de mortalidade infantil é importante principalmente na avaliação da eficácia de tratamentos pediátricos.

Com esta finalidade, foi proposto o índice PIM (Paediatric index of mortality) no trabalho de Shann F. et al (1996), utilizando-se um modelo logístico e um conjunto de dados de pacientes internados em unidades de terapia intensiva na Austrália e na Inglaterra.

A fim de construir um índice de risco de mortalidade infantil adaptado à realidade brasileira, um grupo de estudos do Hospital Universitário da Universidade de São Paulo, 
coordenado pelo Dr. José Carlos Fernandes e Dra. Iracema Fernandes, mediu diversas características em 480 bebês.

De modo análogo ao estudo australiano, o índice de mortalidade infantil brasileiro também foi obtido adotando-se o modelo linear generalizado com funções de ligação logit e probit (McCullagh e Nelder (1989)). As variáveis utilizadas neste estudo são:

Pup: Reação pupilar. Assume o valor 1 se ambas as pupilas têm dilatação de $3 \mathrm{~mm}$ e são fixas e 0 , caso contrário.

Espe: Diagnóstico específico. Pode assumir uma das seguintes categorias:

$1=$ Parada Cardio-pulmonar;

2 = Imunodeficiência grave;

3 = Leucemia ou linfoma antes da primeira indução;

4 = Sangramento do sistema nervoso central;

5 = Miocardite ou miocardiopatia;

6 = Ventrículo esquerdo hipoplásico;

7 = Infecção pelo HIV;

$8=\mathrm{QI}<35$ (menor que o dos pacientes com síndrome de Down);

$9=$ Desordem neurodegenerativa.

Ele: Admissão eletiva. Assume o valor 1 se houve internação e 0, caso contrário.

Vm: Ventilação Mecânica. Indica se o paciente foi intubado na primeira hora de internação.

Be: Base excess.

Pas: Pressão arterial sistólica.

Fio2: Fração inspirada de oxigênio $\left(\mathrm{O}_{2}\right)$. Indica se houve intubação traqueal ou oxitenda.

Pao2: Pressão arterial de oxigênio $\left(\mathrm{O}_{2}\right)$.

$\mathrm{O}$ ajuste dos modelos propostos foi realizado pelo método de máxima verossimilhança utilizando-se o pacote computacional S-Plus. Primeiro, foi utilizada uma amostra de 50 bebês para a estimação dos coeficientes dos modelos. A seguir, cada dado foi incluído 
na amostra seqüencialmente. O logaritmo da verossimilhança preqüencial para o modelo logístico é $-8028,76$ e para o probit é $-35249,50$. A seguir são apresentadas as curvas de calibração. 


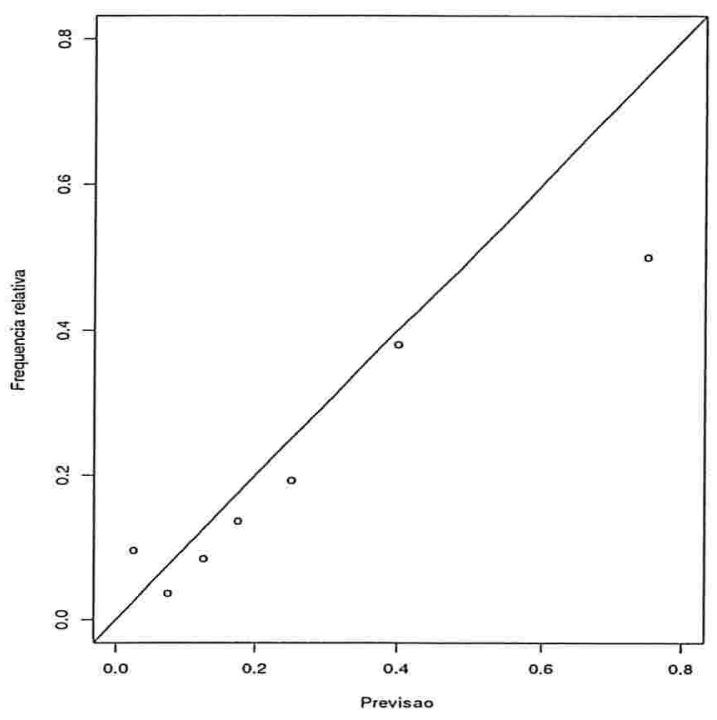

Fig. 2. Curva de calibração para o modelo logit.

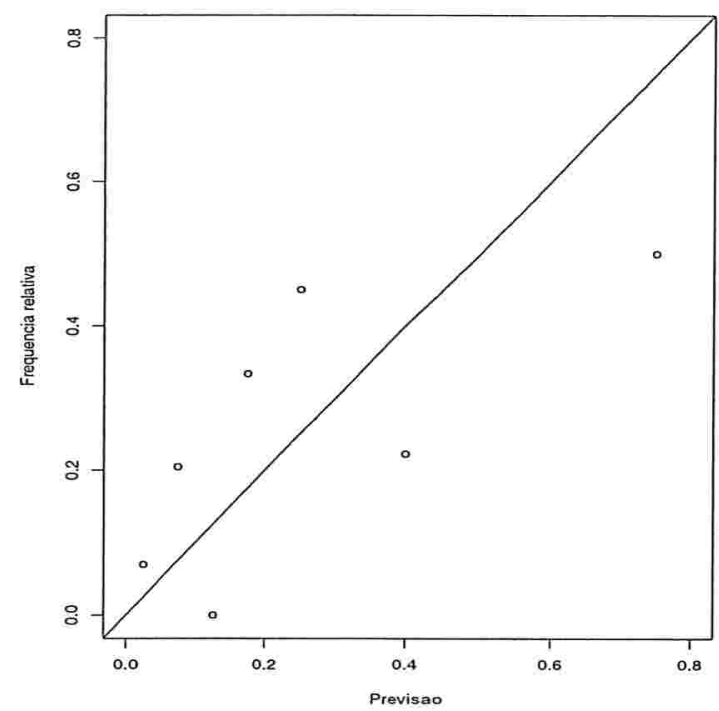

Fig. 3. Curva de calibração para o modelo probit.

A partir desses gráficos e dos valores dos logaritmos da verossimilhança preqüencial podemos concluir que o modelo logístico parece mais apropriado para a construção do índice de mortalidade PIM. 


\subsection{Modelagem de contornos acentuais no português}

Esta aplicação envolve a modelagem de contornos acentuais do português europeu como cadeias de Markov de alta ordem apresentado em Dorea et al. (1997). Neste trabalho são apresentados três métodos de escolha da ordem da cadeia de Markov: os critérios de informação de Akaike e Bayesiano e o critério de mínima entropia.

Um contorno acentual é definido pela seqüência de elementos acentuados e não acentuados. Analisaremos as distâncias entre dois elementos acentuados. Estas distâncias podem assumir valores no espaço de estados $\{1,2,3\}$. Nas tabelas 1,2 e 3 são apresentadas as distribuições de probabilidade estimadas por máxima verossimilhança no trabalho de Dorea et al. (1997) para os contornos acentuais do português europeu, considerando-se as ordens $\ell$ iguais a 0,1 e 2 , respectivamente.

Tabela 1: Distribuição de probabilidade para $\ell=0$.

\begin{tabular}{lccc}
\hline Distância & 1 & 2 & 3 \\
\hline Probabilidade & 0,146 & 0,731 & 0,123 \\
\hline
\end{tabular}

Tabela 2: Probabilidades de transição para $\ell=1$.

\begin{tabular}{cccc}
\hline Distância & 1 & 2 & 3 \\
\hline 1 & 0,289 & 0,658 & 0,053 \\
2 & 0,112 & 0,737 & 0,151 \\
3 & 0,138 & 0,793 & 0,069 \\
\hline
\end{tabular}

A partir destas distribuições de probabilidade, foram simuladas sequiências de tamanho 1000 para cada ordem da cadeia, $\ell=0,1$ ou 2. A simulação foi feita a partir de uma única seqüência de 1000 variáveis uniformemente distribuídas no intervalo [0,1]. A estimação das probabilidades de transição segue o método de máxima verossimilhança. A análise destas seqüências começou com a estimação das probabilidades de transição para os 50 primeiros dados. A seguir, cada dado foi acrescentado na amostra para subseqüente estimação das probabilidades envolvidas e da verossimilhança preqüencial. Nas tabelas 
4 a 6 são apresentados os valores obtidos para a verossimilhança dos modelos, para os coeficientes de informação de Akaike (AIC) e Bayesiano (BIC), entropia $\left(h_{k}\right)$ e o logaritmo da verossimilhança preqüencial (VP). A ordem da cadeia utilizada na simulação será denotada por $\ell$ e os procedimentos de estimação foram calculados supondo-se que a ordem da cadeia seja igual a $k$.

Tabela 3: Probabilidades de transição $\ell=2$.

\begin{tabular}{cccc}
\hline Distâncias & 1 & 2 & 3 \\
\hline 1,1 & 0,333 & 0,583 & 0,083 \\
1,2 & 0,148 & 0,815 & 0,037 \\
1,3 & 0,000 & 1,000 & 0,000 \\
2,1 & 0,238 & 0,762 & 0,000 \\
2,2 & 0,104 & 0,736 & 0,160 \\
2,3 & 0,160 & 0,760 & 0,080 \\
3,1 & 0,400 & 0,400 & 0,200 \\
3,2 & 0,105 & 0,632 & 0,263 \\
3,3 & 0,000 & 1,000 & 0,000 \\
\hline
\end{tabular}

Tabela 4: Estimação da ordem da cadeia para os dados simulados conforme as probabilidades apresentadas na Tabela $1(\ell=0)$.

\begin{tabular}{cccccc}
\hline Order $k$ & $\mathrm{AIC}(k)$ & $\mathrm{BIC}(k)$ & $\mathrm{VP}$ & $\hat{h}_{k}$ & $\mathrm{p}$-value \\
\hline 0 & 1473,35 & 1483,16 & $-692,99$ & 0,7347 & 0,1235 \\
1 & 1474,10 & 1503,55 & $-686,23$ & 0,7318 & 0,9782 \\
2 & 1493,84 & 1582,18 & $-671,21$ & 0,7304 & \\
\hline
\end{tabular}

Tabela 5: Estimação da ordem da cadeia para os dados simulados conforme as probabilidades apresentadas na Tabela $2(\ell=1)$.

\begin{tabular}{cccccc}
\hline Order $k$ & $\mathrm{AIC}(k)$ & $\mathrm{BIC}(k)$ & $\mathrm{VP}$ & $\hat{h}_{k}$ & p-value \\
\hline$k=0$ & 1481,09 & 1490,91 & $-700,29$ & 0,7385 & $<0,0001$ \\
$k=1$ & 1451,63 & 1481,08 & $-678,28$ & 0,7205 & 0,7596 \\
$k=2$ & 1467,31 & 1555,65 & $-665,55$ & 0,7171 & \\
\hline
\end{tabular}


Tabela 6: Estimação da ordem da cadeia para os dados simulados conforme as probabilidades apresentadas na Tabela $3(\ell=2)$.

\begin{tabular}{cccccc}
\hline Order $k$ & $\mathrm{AIC}(k)$ & $\mathrm{BIC}(k)$ & $\mathrm{VP}$ & $\hat{h}_{k}$ & $\mathrm{p}$-value \\
\hline$k=0$ & 1533,80 & 1543,62 & $-732,25$ & 0,7649 & $<0,0001$ \\
$k=1$ & 1504,28 & 1533,73 & $-707,99$ & 0,7469 & $<0,0001$ \\
$k=2$ & 1461,56 & 1549,90 & $-665,00$ & 0,7142 & \\
\hline
\end{tabular}

A ordem da cadeia de acordo com os critérios de Akaike e Bayesiano deve ser escolhida de modo a minimizar os coeficientes AIC e BIC, respectivamente. Outro possível critério é escolher a ordem que minimiza a entropia. Como a entropia do processo cresce com o aumento da ordem da cadeia e a partir de certa ordem converge, existe um teste proposto em Dorea (1997) para avaliar a igualdade de entropias. Assim, a menor ordem para a qual as entropias são iguais é a ordem estimada para a cadeia.

De acordo com os resultados apresentados nas tabelas anteriores, os critérios de Akaike e de mínima entropia apresentam ordem (k) estimada igual à ordem da cadeia $(\ell)$ utilizada na simulação. Segundo o critério Bayesiano, a ordem estimada coincide com a verdadeira ordem quando esta é igual a 0 e 1 e apresenta estimativa igual a 1 quando a verdadeira ordem é 2.

Outro critério semelhante ao de Akaike e Bayesiano poderia ser proposto em função da verossimilhança preqüencial. Em Dawid (1992b), o critério que minimiza a verossimilhança preqüencial se mostra bastante adequado no caso de processos autoregressivos, mas no caso de cadeias de Markov de alta ordem, podemos notar que nos exemplos acima, estas verossimilhanças crescem com o aumento na ordem da cadeia.

\subsection{Sobrevivência após o diagnóstico de mieloma}

Neste estudo, foram estudados os tempos de sobrevivência após o diagnóstico de mieloma de 65 pacientes. Estes dados podem ser encontrados no artigo de Krall et al. (1975). 
Os tempos foram estudados segundo a metodologia de análise de sobrevivência (Kalbfleisch e Prentice (1980)), as análises foram feitas no programa S-Plus. As variáveis explicativas utilizadas na modelagem foram:

- Logaritmo da quantidade de nitrogênio no sangue;

- Medidas de hemoglobina;

- Idade no instante do diagnóstico;

- Sexo;

- Cálcio sérico no instante do diagnóstico.

Foi utilizado o modelo exponencial de modo que a probabilidade do tempo de sobrevivência do indivíduo ser maior que t é $P(T>t)=\exp (\theta t)$.

Os coeficientes do modelo foram estimados de modo seqüencial, começando com uma amostra de 20 indivíduos e posteriormente acrescentando-se cada indivíduo. Após o cálculo do logaritmo dos tempos, o procedimento de estimação é semelhante à metodologia de modelos lineares generalizados por máxima verossimilhança.

A avaliação do sistema de previsão foi baseada na transformada integral de probabilidade. A uniformidade das funções de distribuição pode ser estudada no histograma a seguir.

É interessante notar que este tipo de avaliação do modelo pode ser uma alternativa às análises de resíduos usuais. Além disso, nos casos em que o histograma das funções de distribuição calculadas nos tempos de sobrevivência se desvia muito da distribuição uniforme, este histograma pode ser utilizado para recalibrar as previsões. Após esta recalibração, outras avaliações podem ser feitas a fim de verificar a eficiência da recalibração. 


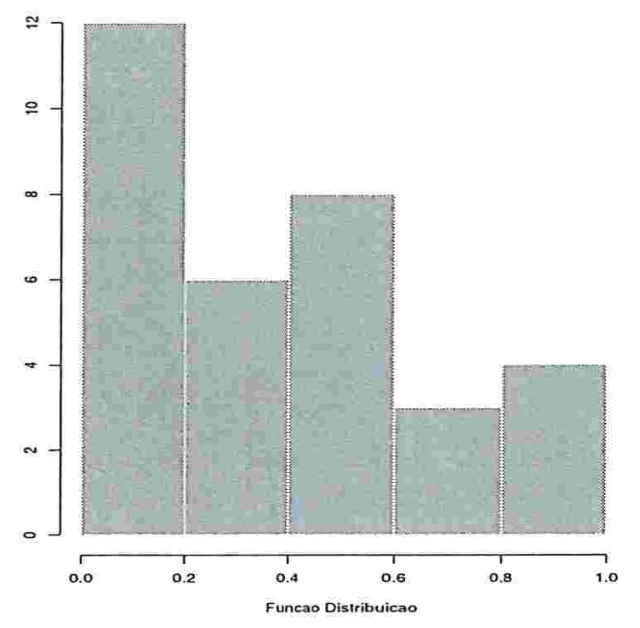

Fig. 4. Histograma das transformadas integrais de probabilidade.

\subsection{Séries temporais com modelos autoregressivos}

A motivação para a análise de séries temporais com modelos autoregressivos surgiu a partir de uma aplicação apresentada em Dawid (1992b). Neste artigo, o autor sugere que o valor da verossimilhança preqüencial é determinante na identificação da ordem de modelos autoregressivos. Utilizando duas séries simuladas de tamanho 500, uma delas de variáveis independentes com distribuição normal padrão e a outra com modelo autoregressivo de ordem 3 , ele consegue identificar a ordem do modelo apenas escolhendo o modelo que maximiza o valor da verossimilhança preqüencial.

No presente trabalho, utilizamos as seguintes séries de tamanho 500:

- Série simulada a partir do modelo $\mathrm{AR}(1): Y_{t}=0,7 Y_{t-1}+e_{t}, \operatorname{com} e_{t} \sim \mathcal{N}(0 ; 0,04)$ independentes;

- Série simulada a partir do modelo $\operatorname{AR}(2): Y_{t}=0,4 Y_{t-1}+0,3 Y_{t-2}+e_{t}$, $\operatorname{com} e_{t} \sim$ $\mathcal{N}(0 ; 0,04)$ independentes; 
- Série correspondente ao logaritmo do retorno das ações da Petrobrás negociadas na Bolsa de Valores de São Paulo, de julho de 1996 a julho de 1998.

Os modelos propostos foram:

- $\operatorname{AR}(1): Y_{t}=\theta_{1} Y_{t-1}+e_{t}, \operatorname{com} e_{t} \sim \mathcal{N}\left(0, \frac{1}{\tau}\right)$ independentes;

- $\operatorname{AR}(2): Y_{t}=\theta_{1} Y_{t-1}+\theta_{2} Y_{t-2}+e_{t}, \operatorname{com} e_{t} \sim \mathcal{N}\left(0, \frac{1}{\tau}\right)$ independentes.

Para a análise dos dados, utilizamos os métodos de previsão "plug-in" e bayesiano. No método "plug-in", os estimadores dos parâmetros dos modelos autoregressivos foram obtidos por máxima verossimilhança. No método bayesiano, as prioris escolhidas foram: $\theta_{1}$ e $\theta_{2}$ independentes com distribuição normal padrão e $\tau$ com distribuição exponencial com média igual a 1. Ao contrário da priori adotada no artigo de Dawid (1992b), é importante ressaltar que a priori aqui escolhida é própria. A escolha da distribuição normal-gama como priori facilitou em parte a obtenção da distribuição preditiva que teve que ser normalizada numericamente.

Para tal, utilizamos o seguinte resultado:

$$
\int_{-\infty}^{\infty} f(y) d y=\int_{-\infty}^{\infty} \frac{f(y)}{g(y)} g(y) d y=\mathbb{E}_{g}\left(\frac{f(Y)}{g(Y)}\right)
$$

em que $g$ corresponde a uma função densidade de probabilidade conhecida. Assim, com uma série de $n$ valores gerados a partir da densidade $g$, a integral acima pôde ser aproximada por $\sum_{i=1}^{n} \frac{f\left(y_{i}\right)}{g\left(y_{i}\right)}$, de acordo com a lei dos grandes números (parte do teorema da representação).

No nosso caso, para obtenção dessa integral, foram gerados 1000 valores da distribuição normal, com média igual à média dos últimos 20 valores da série analisada e variância igual a 0,09. Vale salientar que a utilização destes valores para o cálculo da distribuição preditiva bayesiana não deve ser entendida como uma violação do princípio preqüencial. Primeiro, porque sua utilização constitui um mero artifício numérico de aproximação e, segundo, porque nessa simulação apenas são utilizadas observações anteriores à variável a ser prevista, por meio da média das últimas 20 observações. 
Começando com uma amostra de 50 dados, foram obtidas seqüencialmente as distribuições preditivas para as variáveis $Y_{i}, \operatorname{com} i=51, \ldots, 500$. Tanto pelo método "plug-in", quanto pelo método bayesiano, obtivemos os valores dos logaritmos da verossimilhança preqüencial e as seqüências de distribuições acumuladas até o valor observado correspondente à variável a ser prevista.

As tabelas 7 e 8 apresentam os valores do logaritmo da verossimilhança preqüencial para as três séries consideradas e para os dois modelos adotados, utilizando-se os métodos de previsão "plug-in" e bayesiano, respectivamente.

Tabela 7: Logaritmo da verossimilhança preqüencial para as séries e modelos adotados com método de previsão "plug-in".

\begin{tabular}{lcc}
\hline Série & Modelo AR(1) & Modelo AR(2) \\
\hline $\operatorname{AR}(1)$ & 73,04 & 68,45 \\
$\operatorname{AR}(2)$ & 41,98 & 68,98 \\
Log(retorno das ações) & 841,82 & 839,96 \\
\hline
\end{tabular}

Tabela 8: Logaritmo da verossimilhança preqüencial para as séries e modelos adotados com método de previsão bayesiano.

\begin{tabular}{lcc}
\hline Série & Modelo AR(1) & Modelo AR(2) \\
\hline $\operatorname{AR}(1)$ & 61,68 & 60,51 \\
$\operatorname{AR}(2)$ & 35,84 & 61,31 \\
Log(retorno das ações) & 588,95 & 587,75 \\
\hline
\end{tabular}

A partir das tabelas acima, podemos confirmar que, para cada série autoregressiva, a verossimilhança preqüencial é maximizada quando a ordem do modelo adotado coincide com a verdadeira ordem da série. Assim, a escolha da ordem do modelo, segundo o critério de maximização da verossimilhança preqüencial, parece bastante confiável, o que confirma o resultado apresentado em Dawid (1992b).

Para a série de ações, podemos verificar que para os dois métodos de previsão a ordem 1 seria escolhida. Além disso, os valores da verossimilhança preqüencial são bem maiores, mas isto se deve somente à maior variabilidade dessa série. 
Além da verificação do critério de máxima verossimilhança preqüencial, construímos histogramas das seqüências de distribuições preditivas acumuladas até o valor efetivamente observado. Conforme o resultado apresentado na seção 4.5, estas seqüências devem apresentar distribuição uniforme se os modelos propostos forem adequados ao objetivo preditivo. Deste modo, para um modelo apropriado, os histogramas devem apresentar forma semelhante à distribuição uniforme em [0,1].

A seguir, são apresentados os histogramas das seqüências de distribuições acumuladas para as séries $A R(1)$ com os modelos $A R(1)$ e $A R(2)$ e das ações com modelo $A R(1)$, utilizando-se o método bayesiano de previsão.

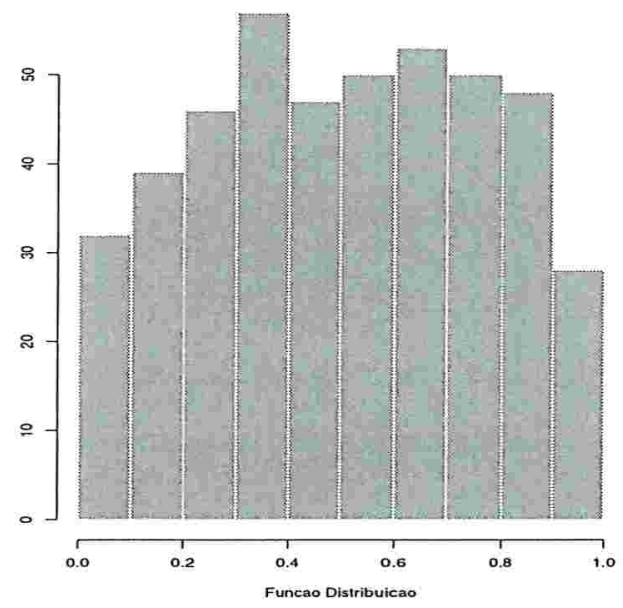

Fig. 5. Histograma das transformadas integrais de probabilidade para a série $A R(1)$ com modelo $A R(1)$. 


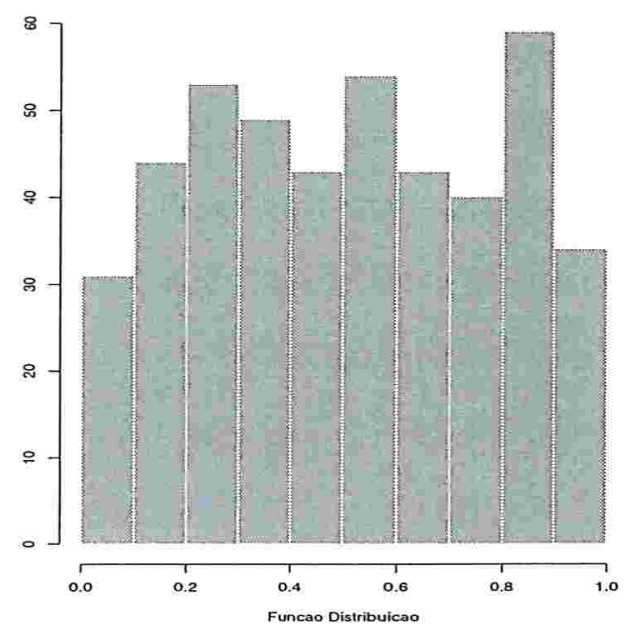

Fig. 6. Histograma das transformadas integrais de probabilidade para a série $A R(1)$ com modelo $A R(\mathcal{Q})$.

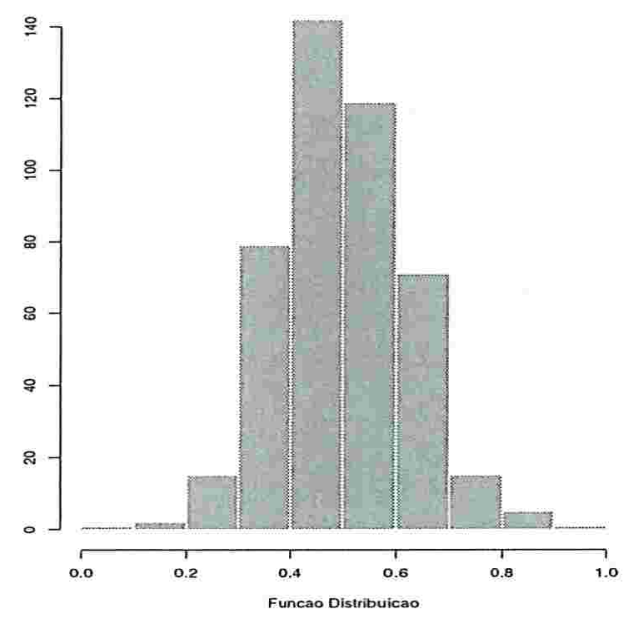

Fig. 7. Histograma das transformadas integrais de probabilidade para a série do logaritmo do retorno das ações com modelo $A R(1)$.

Podemos verificar que não basta analisarmos somente estes histogramas para escolhermos a ordem do processo, pois os histogramas para a série $A R(1)$ com os modelos $A R(1)$ 
e $\mathrm{AR}(2)$ são muito parecidos, o que inviabiliza uma escolha entre os dois modelos, ao contrário do que ocorre com o critério da verossimilhança preqüencial.

Além disso, não basta adotarmos um modelo autoregressivo para construir um sistema estatístico de previsão e utilizarmos critérios como o da verossimilhança preqüencial a fim de escolhermos a ordem do processo. Outro método de avaliação deve ser empregado para avaliar se modelos autoregressivos são apropriados. De acordo com o histograma para a série de ações, podemos observar que o modelo autoregressivo de ordem 1 não é adeqüado para esta série. 


\section{Capítulo 8}

\section{Conclusões}

A análise preqüencial pode ser entendida como uma nova abordagem do problema de inferência estatística. Diversos problemas podem ser estudados sob a ótica preqüencial, incluindo também diversos estudos que não sejam necessariamente de natureza seqüencial e preditiva.

A ênfase na necessidade de avaliação dos métodos de previsão é um aspecto essencial da análise preqüencial. Além disso, o embasamento de tais avaliações nas previsões produzidas pelos sistemas de previsão e em quantidades efetivamente observadas, como enunciado no Princípio Preqüencial, expressa de modo exemplar a visão operacionalista aplicada à inferência estatística.

As aplicações apresentadas ilustram como a análise preqüencial sugere uma alternativa à análise de resíduos usuais, e como pode também ser utilizada para corrigir modelos que não apresentam bom desempenho, propondo a recalibração.

Alguns estudos baseados em simulação, como os que fizemos para modelos autoregressivos e para cadeias de Markov de alta ordem, podem ser futuramente mais explorados visando a confirmação de critérios baseados na verossimilhança preqüencial. 


\section{Referências Bibliográficas}

[1] Arruda, M. L. (1999) Poisson, Bayes, Futebol e De Finetti. Dissertação de Mestrado. IME-USP.

[2] Blackwell, D. e Dubins, L. E. (1962) Merging of opinions with increasing information, Ann. Math. Statist., 33, 882-886.

[3] Brier, G. W. (1950) Verification of forecasts expressed in terms of probability, Monthly Weather Review, 78(1), 1-3.

[4] Dawid, A. P. (1982) The well-calibrated bayesian. Journal of the American Statistical Association, 77 (379), 605-613.

[5] Dawid, A. P. (1984) Statistical theory: the prequential approach. Journal of Royal Statistical Society A, 147, 278-292.

[6] Dawid A. P. (1985) The Impossibility of Inductive Inference. Journal of the American Statistical Association. 80 (390), 340-341.

[7] Dawid, A. P. (1986) Probability forecasting. Encyclopedia of Statistical Sciences, eds S. Kotz, N. L. Johnson e C. B. Read, New York: Wiley-Interscience, 7, 210-218.

[8] Dawid, A. P. (1992a) Prequential Analysis, Stochastic Complexity and Bayesian Inference. Bayesian statistics 4: Proceedings of the Fourth Valencia International Meeting, ed. J. M. Bernardo, J. O. Berger, A. P. Dawid e A. F. M. Smith, Oxford University Press, 109-125. 
[9] Dawid, A. P. (1992b) Prequential data analysis. Current Issues in Statistical Inference: Essays in Honor of D. Basu, IMS Lecture Notes - Monograph Ser. 17. Hayward, CA: Institute of Mathematical Statistics, 113-126.

[10] Dawid, A. P. (1997) Prequential analysis. Encyclopedia of Statistical Sciences - Update, eds S. Kotz, C. B. Read e D. L. Banks, New York: Wiley-Interscience, vol. 1, $464-470$.

[11] Dawid, A. P. e Vovk, V. G. (1999) Prequential probability: principles and properties. Bernoulli, 5(1), 125-162.

[12] Dorea, C., Galves, A., Kira, E. e Alencar, A. P. (1997) Markovian modeling of the stress contours of Brazilian and European Portuguese, Brazilian Journal of Probability and Statistics - REBRAPE, 11(2), 161-173.

[13] Esteves, L. G. (1997) Coincidência de distribuições através de uma transformação de De Finetti. Dissertação de mestrado. IME-USP.

[14] de Finetti, B. (1931) Sul Significato Soggetivo della Probabilitá. Fundamenta Mathematicae, vol. 17, 298-329.

[15] de Finetti, B. (1972) Probability, Induction and Statistics, John Wiley \& Sons.

[16] de Finetti, B. (1974) Theory of Probability, vol.1, London: Wiley.

[17] Goldstein, M. (1985) Temporal Coherence. Bayesian Statistical 2, J. M. Bernardo, M. H. DeGroot, D. V. Lindley e A. F. M. Smith (ed.). Elsevier Science Publishers B. V., North Holland, 231-248.

[18] Kalbfleisch, J. D. e Prentice, R. L. (1980) The Statistical Analysis of Failure Time Data. John Wiley and Sons.

[19] Krall, J. M., Uthoff, V. A. e Harley, J. B. (1975) A step-up procedure for selecting variables associated with survival, Biometrics, 31(1), 49-57. 
[20] Loschi, R. H. (1992) Coerência e Probabilidade. Dissertação de Mestrado. IME-USP.

[21] Loschi, R. H., Iglesias, P. L. e Wechsler, S. (1998) Unpredictability and Probability Updating. São Paulo, IME-USP, 1998, 11p. RT-MAE-9818. Submetido para publicação no volume Bayesian Statistics 6. Em fase de apreciação.

[22] McCullagh, P. and Nelder, J.R. (1989). Generalized Linear Models. 2. ed. London: Chapman and Hall.

[23] Morettin, P. A. e Tolói, C. M. C. (1987) Previsão de séries temporais. São Paulo: Atual.

[24] Murphy, A. H. e Epstein, E. S. (1967) Verification of Probabilistic Predictions: A Brief Review. Journal of Applied Meteorology, 6, 748-755.

[25] Murphy, A. H. (1972a) Scalar and Vector Partitions of the Probability Score: Part I: Two-State Situation. Journal of Applied Meteorology, 11, 273-282.

[26] Murphy, A. H. (1972b) Scalar and Vector Partitions of the Probability Score: Part II: N-State Situation. Journal of Applied Meteorology, 11, 1183-1192.

[27] Murphy, A. H. e Winkler, R. L. (1977) Reliability of Subjective Probability Forecasts of Precipitation and Temperature. Applied Statistics, 26, 41-47.

[28] Oakes, D. (1985) Self-calibrating priors do not exist. Journal of the American Statistical Association, 80, 339-342.

[29] Sanders, F. (1958) The evaluation of subjective probability forecasts. Cambridge, MIT. Department of Meteorology, Contract AF(604)-1305, Tech. Rept. n. 5, 62 pp.

[30] Shann, F., Pearson, G., Slater, A. e Wilkinson, K. (1997) Paediatric index of mortality prediction model for children in intensive care. Intensive Care Med., SpringerVerlag, 23, 201-207.

[31] Shiryayev, A. N. (1984) Probability. New York: Springer-Verlag. 
[32] Smith, A. F. M. (1988) Schwartz criterion. Encyclopedia of Statistical Sciences, eds S. Kotz, N. L. Johnson e C. B. Read, New York: Wiley-Interscience, 8, 289-290.

[33] Stone, M. (1974) Cross-validation choice and assessment of statistical predictions. Journal of Royal Statistical Society B, 36, 111-147.

[34] Wechsler, S. (1993) Exchangeability and Predictivism. Erkenntnis, 38, 343-350.

[35] Zuazola, P. L. I. (1993) Formas finitas do teorema de Finetti: a versão preditivista da inferência estatística em populações finitas. Dissertação de doutorado. IME-USP. 